\title{
A GEOMETRIC CRITERION FOR GELFAND PAIRS ASSOCIATED WITH THE HEISENBERG GROUP
}

\author{
Chal Benson, Joe Jenkins, Ronald L. Lipsman and Gail Ratcliff
}

Let $K$ be a closed subgroup of $U(n)$ acting on the $(2 n+1)$ dimensional Heisenberg group $H_{n}$ by automorphisms. One calls $\left(K, H_{n}\right)$ a Gelfand pair when the integrable $K$-invariant functions on $H_{n}$ form a commutative algebra under convolution. We prove that this is the case if and only if the coadjoint orbits for $G:=K \ltimes H_{n}$ which meet the annihilator $\mathfrak{k}^{\perp}$ of the Lie algebra $\mathfrak{k}$ of $K$ do so in single $K$-orbits. Equivalently, the representation of $K$ on the polynomial algebra over $\mathbb{C}^{n}$ is multiplicity free if and only if the moment map from $\mathbb{C}^{n}$ to $\mathfrak{k}^{*}$ is one-to-one on $K$-orbits.

It is also natural to conjecture that the spectrum of the quasi-regular representation of $G$ on $L^{2}(G / K)$ corresponds precisely to the integral coadjoint orbits that meet $\mathfrak{k}^{\perp}$. We prove that the representations occurring in the quasi-regular representation are all given by integral coadjoint orbits that meet $\mathfrak{k}^{\perp}$. Such orbits can, however, also give rise to representations that do not appear in $L^{2}(G / K)$.

\section{Introduction.}

Let $V \cong \mathbb{C}^{n}$ be a finite dimensional complex vector space with Hermitian inner product $\langle\cdot, \cdot\rangle$. We denote the real and imaginary parts of $\langle\cdot, \cdot\rangle$ by $(\cdot, \cdot)$ and $\omega(\cdot, \cdot)$ so that

$$
\langle\cdot, \cdot\rangle=(\cdot, \cdot)+i \omega(\cdot, \cdot) .
$$

The bilinear forms $(\cdot, \cdot)$ and $\omega(\cdot, \cdot)$ are a positive definite inner product and a symplectic structure respectively on the underlying real vector space $V_{\mathbb{R}} \cong$ $\mathbb{R}^{2 n}$ of $V$. The associated Heisenberg group $H_{V} \cong H_{n}$ is

$$
H_{V}:=V \times \mathbb{R} \quad \text { with product } \quad(z, t)\left(z^{\prime}, t^{\prime}\right):=\left(z+z^{\prime}, t+t^{\prime}-\frac{1}{2} \omega\left(z, z^{\prime}\right)\right) .
$$

This is a real 2-step nilpotent Lie group of dimension $2 n+1$ with center $\{0\} \times \mathbb{R}$. 
Let $K$ be a compact Lie subgroup of $\operatorname{Aut}\left(H_{V}\right)$. We say that $\left(K, H_{V}\right)$ is a Gelfand pair when the subalgebra $L_{K}^{1}\left(H_{V}\right)$ of $K$-invariant elements in $L^{1}\left(H_{V}\right)$ is commutative under convolution. Equivalently, the $K$-bi-invariant $L^{1}$-functions on

$$
G:=K \ltimes H_{V}
$$

form a commutative algebra. We will prove below in Section 2.1 that $\left(K, H_{V}\right)$ is a Gelfand pair if and only if $\left(K_{\circ}, H_{V}\right)$ is a Gelfand pair where $K_{\circ}$ is the identity component in $K$. Moreover, if $\left(K, H_{V}\right)$ is a Gelfand pair then so is $\left(K^{\prime}, H_{V}\right)$ for any conjugate $K^{\prime}$ of $K$ inside $\operatorname{Aut}\left(H_{V}\right)$ [2]. The unitary group $U(V) \cong U(n)$ of automorphisms of $V$ preserving $\langle\cdot, \cdot\rangle$ embeds in $\operatorname{Aut}\left(H_{V}\right)$ via

$$
k \cdot(z, t):=(k z, t)
$$

and yields a maximal compact connected subgroup of Aut $\left(H_{V}\right)$. These observations allow us to restrict attention to closed connected subgroups of $U(V)$.

The following result summarizes some conditions that are known to be equivalent to $\left(K, H_{V}\right)$ being a Gelfand pair. A unitary representation is said to be multiplicity free if its isotypic components are irreducible. The action of $K$ on $V$ is said to be a multiplicity free action when the representation of $K$ on $\mathbb{C}[V]$ given by $(k \cdot p)(z):=p\left(k^{-1} z\right)$ is multiplicity free. Equivalently, the complexified representation of $K_{c}$ on $\mathbb{C}[V]$ is multiplicity free.

Theorem 1.1. Let $K$ be a closed connected subgroup of $U(V)$. The following conditions are equivalent:

(1) $\left(K, H_{V}\right)$ is a Gelfand pair.

(2) For all $\pi$ in the unitary dual $\widehat{G}=\widehat{K \ltimes H}_{V}$, the multiplicity of the trivial representation $1_{K}$ in $\left.\pi\right|_{K}$ is 0 or 1 .

(3) The quasi-regular representation $\operatorname{Ind}_{K}^{G}\left(1_{K}\right)$ of $G$ on $L^{2}(G / K) \cong$ $L^{2}\left(H_{V}\right)$ is multiplicity free.

(4) For all $\pi \in \widehat{H_{V}}$, the representation $W_{\pi}$ of

$$
K_{\pi}:=\{k \in K: \pi \circ k \text { is unitarily equivalent to } \pi\}
$$

on the representation space $\mathcal{H}_{\pi}$ of $\pi$ by intertwining operators is multiplicity free.

(5) The action of $K$ on $V$ is a multiplicity free action.

(6) A Borel subgroup in $K_{c}$ has a Zariski-open orbit in $V$. 
(7) The algebra $\mathcal{P} \mathcal{D}(V)^{K}=\mathcal{P} \mathcal{D}(V)^{K_{c}}$ of $K$-invariant polynomial coefficient differential operators on $V$ is abelian.

The equivalence of (1) and (2) is a special case of a standard result due to I.M. Gelfand [9]. Conditions (2) and (3) are equivalent by Ahn reciprocity [23, pp. 56-58]. One uses the Mackey machine for $\widehat{G}$ to show that $(2)$ and (4) are equivalent $[2,4]$. In fact, it suffices that (4) hold for a.e. $\pi \in \widehat{H_{V}}[2]$. One can show that conditions (5) and (4) are equivalent by using the Fock model for the generic representations of $H_{V}$ [2]. The equivalence of (5) and (6) can be found, for example, in [16], and $(6) \Leftrightarrow(7)$ is proved in [15].

Remark 1.1. Conditions (2), (3) and (4) are equivalent to $\left(K, H_{V}\right)$ being a Gelfand pair for any compact subgroup $K \subset \operatorname{Aut}\left(H_{V}\right)$. Also, (5) and (1) are equivalent even when $K$ fails to be connected. We use these facts later in the proof of Proposition 2.2.

We wish to develop criteria for Gelfand pairs which involve the geometry of coadjoint orbits. Representations of some classes of Lie groups may be obtained via the orbit method (also called geometric quantization), a process which begins with (integral) coadjoint orbits. For example, this method describes the unitary dual of compact groups [10, 12], nilpotent groups (where it reduces to the usual Kirillov correspondence [17]) and of semidirect products of nilpotent groups by compact groups [24]. In particular, there is a correspondence between irreducible unitary representations of $G=K \ltimes H_{V}$ and integral coadjoint orbits in the dual $\mathfrak{g}^{*}$ of the Lie algebra $\mathfrak{g}$ of $G$.

Known geometric multiplicity formulae take the following general form: Given a subgroup $H$ of the Lie group $L$, let $\pi_{\circ} \in \widehat{H}$ and $\pi \in \widehat{L}$ correspond to coadjoint orbits $\mathcal{O}_{\circ} \subset \mathfrak{h}^{*}$ and $\mathcal{O} \subset \mathfrak{r}^{*}$ respectively. Let $p: \mathfrak{l}^{*} \rightarrow \mathfrak{h}^{*}$ be the restriction map. Then it should follow that the multiplicity of $\pi_{\circ}$ in $\left.\pi\right|_{H}$ or of $\pi$ in $\operatorname{Ind}_{H}^{G}\left(\pi_{\circ}\right)$ is $\#\left(\mathcal{O} \cap p^{-1}\left(\mathcal{O}_{\circ}\right)\right) / H$, the number of $H$-orbits in $\mathcal{O}$ which restrict to $\mathcal{O}_{\circ}$. Such formulae appear in direct integral decompositions for nilpotent groups $[\mathbf{5}, \mathbf{6}, \mathbf{7}, \mathbf{2 5}]$, completely solvable and exponentially solvable groups $[\mathbf{2 5}, \mathbf{2 6}]$. It is also known to hold "asymptotically" for representations of compact groups $[\mathbf{1 0}, \mathbf{1 2}]$ and to some extent for Riemannian symmetric spaces [25].

Consider the group $G=K \ltimes H_{V}$ with Lie algebra $\mathfrak{g}=\mathfrak{k} \ltimes \mathfrak{h}_{V}$ and restriction map $p: \mathfrak{g}^{*} \rightarrow \mathfrak{k}^{*}$. The coadjoint orbit corresponding to $1_{K} \in \widehat{K}$ is $\{0\}$ and $p^{-1}(\{0\})=\mathfrak{k}^{\perp} \cong \mathfrak{h}_{V}^{*}$. One should hope that representations $\pi$ of $G$ occurring in $\operatorname{Ind}_{K}^{G}\left(1_{K}\right)$ (equivalently those whose restriction to $K$ contains $1_{K}$ ) correspond to integral coadjoint orbits in $\mathfrak{g}^{*}$ which meet $\mathfrak{k}^{\perp}$. Moreover, one expects the multiplicity of $1_{K}$ in $\left.\pi\right|_{K}$ to be the number of $K$-orbits in the intersection $\mathcal{O} \cap \mathfrak{k}^{\perp}$ where $\mathcal{O} \subset \mathfrak{g}^{*}$ is the coadjoint orbit for $\pi$. Denote the 
coadjoint orbit in $\mathfrak{g}^{*}$ through $\xi \in \mathfrak{g}^{*}$ by $\mathcal{O}_{\xi}^{G}$. Then, in view of the equivalence of conditions (1) and (2) in Theorem 1.1, the preceding discussion suggests the following theorem, which is our main result.

Theorem 1.2. Let $K$ be a compact connected subgroup of $U(V)$. Then $\left(K, H_{V}\right)$ is a Gelfand pair if and only if

$$
\text { for every } \xi \in \mathfrak{k}^{\perp}, \mathcal{O}_{\xi}^{G} \cap \mathfrak{k}^{\perp} \text { is a single } K \text {-orbit. }
$$

A closely related result, due to V. Guillemin and S. Sternberg, concerns compact group actions on cotangent bundles [11]. In fact, [11] contains a geometric characterization of compact Gelfand pairs. Suppose that $L$ is a compact connected Lie group and that $K$ is a closed Lie subgroup of $L$. In this setting, $K \subset L$ is a Gelfand pair ${ }^{1}$ if and only if $\mathcal{O}_{\xi}^{L} \cap \mathfrak{k}^{\perp}$ is a finite union of $K$-orbits for generic integral points $\xi \in \mathfrak{k}^{\perp}$. Our proof of Theorem 1.2, given in Section 5, involves different methods from those in [11], since $G:=K \ltimes H_{V}$ is not compact.

We say that $\xi \in \mathfrak{g}^{*}$ is integral when $\mathcal{O}_{\xi}^{G}$ is an integral coadjoint orbit and write $\rho_{\xi}$ for the corresponding representation of $G$. Integrality means that the map $G_{\xi} \rightarrow \mathbb{T}$ defined by $\exp (X) \mapsto e^{i \xi(X)}$ is a unitary character on the stabilizer $G_{\xi}$ of $\xi$ under the coadjoint action. The set of irreducible representations weakly contained in a given unitary representation is called its spectrum. Another natural conjecture is the following:

(SC) The spectrum of $\operatorname{Ind}_{K}^{G}\left(1_{K}\right)$ is $\left\{\rho_{\xi} \mid \xi\right.$ is an integral point in $\left.\mathfrak{k}^{\perp}\right\}$.

Indeed, the orbit method folklore discussed above motivates this "guess". We will show that $\left\{\rho_{\xi} \mid \xi\right.$ is an integral point in $\left.\mathfrak{k}^{\perp}\right\}$ always contains the spectrum of $\operatorname{Ind}_{K}^{G}\left(1_{K}\right)$. (See Proposition 4.1.) We will, however, present examples showing that this containment can be proper, even when $\left(K, H_{V}\right)$ is a Gelfand pair. (See Examples 4.1 and 7.2.) Characterizing the situations where Condition (SC) holds seems to be an interesting but difficult problem.

In Section 3, we reformulate conditions (OC) and (SC) in terms of the actions of $K$ on $V$ and $\mathbb{C}[V]$. A key ingredient is the (un-normalized) moment map $\tau: V \rightarrow \mathfrak{k}^{*}$ for the action of $K$ on $V$ defined by

$$
\tau(z)(A):=\omega(z, A z) \quad \text { for } z \in V, A \in \mathfrak{k} .
$$

It is easy to see that $\tau$ is $K$-equivariant. We show in Theorem 3.1 that Condition (OC) holds if and only if $\tau$ is injective on $K$-orbits. In Theorem 3.2,

\footnotetext{
${ }^{1}$ i.e. the $K$-bi-invariant $L^{1}$-functions on $L$ form a commutative convolution algebra
} 
we show that Condition (SC) holds for $\left(K, H_{V}\right)$ if and only if the spectrum of the representation of $K$ on $\mathbb{C}[V]$ is given precisely by the integral coadjoint orbits which lie in $\tau(V) \subset \mathfrak{k}^{*}$. These results together with Condition (5) in Theorem 1.1 enable one to remove the semidirect product $G=K \ltimes H_{V}$ from the picture. All of this rests on a description of the coadjoint orbits in $G$ derived in Section 2.5. The reformulated version of Theorem 1.2 reads:

Theorem 1.3. The action of $K$ on $V$ is multiplicity free if and only if $\tau$ is one-to-one on K-orbits.

The condition in Theorem 1.3 means that if $\tau\left(K v_{1}\right)=\tau\left(K v_{2}\right)$ then $K v_{1}=$ $K v_{2}$. Equivalently, $\tau^{-1}\left(\mathcal{O}_{\alpha}^{K}\right)$ is at most a single $K$-orbit for each $\alpha \in \mathfrak{k}^{*}$. In the language of symplectic geometry, the Marsden-Weinstein reduction of $V$ at $\alpha$ is a single point for each $\alpha \in \tau(V)$.

The moment map induces a map $\tau^{*}: I\left[\mathfrak{k}^{*}\right] \rightarrow \mathbb{C}\left[V_{\mathbb{R}}\right]^{K}$ from the $\operatorname{Ad}^{*}(K)$ invariant polynomials $I\left[\mathfrak{k}^{*}\right]$ on $\mathfrak{k}^{*}$ to the $K$-invariant polynomials $\mathbb{C}\left[V_{\mathbb{R}}\right]^{K}$ on $V_{\mathbb{R}}$. Our proof of Theorem 1.3 in Section 5 rests on the fact that the multiplicity free condition and injectivity of $\tau$ on $K$-orbits are both equivalent to algebraic conditions involving the ring inclusion $\tau^{*}\left(I\left[\mathfrak{k}^{*}\right]\right) \subset \mathbb{C}\left[V_{\mathbb{R}}\right]^{K}$. In particular, the fraction field of $\mathbb{C}\left[V_{\mathbb{R}}\right]^{K}$ must be a finite extension of the fraction field of $\tau^{*}\left(I\left[\mathfrak{k}^{*}\right]\right)$. (See Propositions 5.3 and 5.4.)

The condition concerning fraction fields certainly holds whenever $\tau^{*}$ is surjective. We say that the action of $K$ on $V$ is a Capelli action in this case. In Section 6 we show that an action is Capelli if and only if the "abstract Capelli problem" for its complexification (introduced in [15]) has an affirmative answer. Capelli actions provide a special class of multiplicity free actions. The algebraic conditions established in Section 5 show that a general multiplicity free action is "almost" a Capelli action. We examine several examples of Capelli actions more closely in Section 7, considering Condition (SC) in each case.

Variants of the main results in this paper were announced in [1]. Theorems 1.2 and 1.3 appear in [1] as conjectures. In fact, Theorem 1.3 can be obtained from the somewhat weaker result in [1] by appealing to a result, due to Lerman, Montgomery and Sjamaar, concerning connectivity of the fibers of the moment map for a compact linear action. This is explained below in Section 5. We are indebted to Yael Karshon for drawing our attention to the paper [21].

1.1. Notation. Throughout this paper, $H_{V}$ will denote the Heisenberg group given by a Hermitian vector space $(V,\langle\cdot, \cdot\rangle)$ of complex dimension $n$. $K$ will denote a compact Lie subgroup of $\operatorname{Aut}\left(H_{V}\right)$. After Section 2.1, $K$ will always be a connected subgroup of $U(V)$ unless stated otherwise. $G$ 
denotes the semidirect product $G=K \ltimes H_{V}$. The Lie algebras of $H_{V}, K$ and $G$ are $\mathfrak{h}_{V}, \mathfrak{k}$ and $\mathfrak{g} . \mathfrak{k}_{c}=\mathfrak{k} \otimes \mathbb{C}$ is the Lie algebra of the complexification $K_{c}$ of $K$. For $\gamma$ in the linear duals $\mathfrak{k}^{*}, \mathfrak{g}^{*}$ or $\mathfrak{h}_{V}^{*}, \mathcal{O}_{\gamma}^{K}, \mathcal{O}_{\gamma}^{G}$ and $\mathcal{O}_{\gamma}^{H_{V}}$ denote $K-, G$ and $H_{V^{-}}$-coadjoint orbits respectively through $\gamma \cdot \mathfrak{k}^{\perp} \subset \mathfrak{g}^{*}$ is the annihilator of $\mathfrak{k}$ in $\mathfrak{g}^{*} . \mathbb{C}[V]$ denotes the algebra of holomorphic polynomials on the complex vector space $V$ and $\mathbb{C}\left[V_{\mathbb{R}}\right]$ are the polynomials on the underlying real vector space $V_{\mathbb{R}} \cdot \mathbb{C}\left[V_{\mathbb{R}}\right]^{K}$ denotes the algebra of $K$-invariant polynomials for the contragredient action of $K$ on $\mathbb{C}\left[V_{\mathbb{R}}\right] . I[\mathfrak{k}]$ and $I\left[\mathfrak{k}^{*}\right]$ denote the algebras of $\operatorname{Ad}(K)$ - and $\operatorname{Ad}^{*}(K)$-invariant complex valued polynomials on the real vector spaces $\mathfrak{k}$ and $\mathfrak{k}^{*}$.

1.2. Acknowledgment. This work has benefited greatly from conversations and correspondence with other mathematicians. We especially wish to thank Roger Howe, Yael Karshon, Friedrich Knop and Prabhakar Rao for their helpful suggestions.

\section{Preliminaries.}

2.1. Connectivity of $K$. The following Lemma shows that the notion of Gelfand pair cannot be generalized by replacing the "multiplicity free" criterion by a "bounded multiplicity" condition. This result has a folklore status among researchers. We learned of it from Frederic Bien and Roger Howe on separate occasions and a related result appears in [20]. We include a brief proof for the reader's convenience.

Lemma 2.1. Let $K \subset U(V)$ be connected. If $\left(K, H_{V}\right)$ is not a Gelfand pair then $\mathbb{C}[V]$ contains irreducible $K$-modules with arbitrarily large multiplicities.

Proof. Let $\mathbb{C}[V]=\sum_{\alpha} \mathcal{P}_{\alpha}$ be a decomposition of $\mathbb{C}[V]$ into $K$-irreducibles. Since $\left(K, H_{V}\right)$ is not a Gelfand pair, there are two factors, $\mathcal{P}_{\alpha}$ and $\mathcal{P}_{\beta}$ say, which are equivalent as $K$-modules. Let $p_{\alpha}$ and $p_{\beta}$ denote highest weight vectors in $\mathcal{P}_{\alpha}$ and $\mathcal{P}_{\beta}$ with common highest weight $\chi$ on a maximal torus $T \subset K$. By removing any common prime factors, we can assume that $p_{\alpha}$ and $p_{\beta}$ are relatively prime. For any fixed integer $N>0, p_{\alpha}^{k} p_{\beta}^{N-k}$ is a highest weight vector with weight $\chi^{N}$ for each $k=0,1, \ldots, N$. We claim that $\left\{p_{\alpha}^{k} p_{\beta}^{N-k} \mid k=0,1, \ldots, N\right\}$ is a linearly independent set in $\mathbb{C}[V]$. Otherwise we could write some $p_{\alpha}^{k_{\circ}} p_{\beta}^{N-k_{\circ}}$ as a linear combination

$$
p_{\alpha}^{k_{\circ}} p_{\beta}^{N-k_{\circ}}=\sum_{k=k_{\circ}+1}^{N} c_{k} p_{\alpha}^{k} p_{\beta}^{N-k}
$$

and conclude that $p_{\alpha}^{k_{\circ}+1}$ divides $p_{\alpha}^{k_{\circ}} p_{\beta}^{N-k_{\circ}}$. This is impossible since $p_{\alpha}$ and $p_{\beta}$ are relatively prime. Thus, the $K$-modules generated by $p_{\alpha}^{k} p_{\beta}^{N-k}$ for 
$k=0,1, \ldots, N$ are pair-wise distinct copies in $\mathbb{C}[V]$ of a single $K$-irreducible.

The unitary automorphisms $U(V)$, given by Equation 1.4, together with the flip automorphism $f: H_{V} \rightarrow H_{V},(z, t) \mapsto(\bar{z},-t)$ generate a maximal compact subgroup $M$ of $\operatorname{Aut}\left(H_{V}\right){ }^{2}$ Let $K$ be a (possibly disconnected) compact subgroup of $\operatorname{Aut}\left(H_{V}\right)$. We can assume that $K \subset M$ since the Gelfand pair property is invariant under conjugation inside $\operatorname{Aut}\left(H_{V}\right)$ [2]. The orbit picture for $\widehat{H_{V}}$ makes it clear that $K_{\pi}=K \cap U(V)$ for the generic (infinite dimensional) representations $\pi \in \widehat{H_{V}}$. Thus, Condition (4) in Theorem 1.1 will hold for $K$ if and only if it holds for $K \cap U(V)$. That is, $\left(K, H_{V}\right)$ is a Gelfand pair if and only if $\left(K \cap U(V), H_{V}\right)$ is a Gelfand pair.

Proposition 2.2. Let $K$ be a compact subgroup of $\operatorname{Aut}\left(H_{V}\right)$ with identity component $K_{\circ}$. Then $\left(K, H_{V}\right)$ is a Gelfand pair if and only if $\left(K_{\circ}, H_{V}\right)$ is a Gelfand pair.

Proof. Since $L_{K}^{1}\left(H_{V}\right) \subset L_{K_{\circ}}^{1}\left(H_{V}\right)$ it is clear that $\left(K, H_{V}\right)$ is a Gelfand pair whenever $\left(K_{\circ}, H_{V}\right)$ is a Gelfand pair. On the other hand, suppose that $\left(K, H_{V}\right)$ is a Gelfand pair. As explained above, we can assume that $K \subset M$ and even $K \subset U(V)$. Let

$$
\mathbb{C}[V]=\sum_{\alpha} \mathcal{P}_{\alpha}
$$

denote the (multiplicity free) decomposition of $\mathbb{C}[V]$ into $K$-irreducibles.

Suppose that $K$ has $\ell$ components and let $k_{1}, \ldots, k_{\ell} \in K$ give a complete set of coset representatives for the $K_{\circ}$-cosets in $K$. Suppose that $\left(K_{\circ}, H_{V}\right)$ is not a Gelfand pair. Lemma 2.1 shows that for any $N \geq 1$, we can find an irreducible $K_{\circ}$-module $\rho$ with multiplicity at least $N \ell 2^{\ell}$. Since the $\mathcal{P}_{\alpha}$ 's are $K_{\circ}$-invariant, each of these copies of $\rho$ is contained in some $\mathcal{P}_{\alpha}$. Suppose that $W$ is a copy of $\rho$ inside $\mathcal{P}_{\alpha}$. Then $k_{i} W \subset \mathcal{P}_{\alpha}$ is $K_{\circ}$-invariant (as $K_{\circ}$ is normal in $K$ ) and equivalent to $\rho_{k_{i}} \in \widehat{K_{\circ}}$ defined by

$$
\rho_{k_{i}}(k)=\rho\left(k_{i} k k_{i}^{-1}\right) .
$$

Since $\mathcal{P}_{\alpha}$ is $K$-irreducible,

$$
\mathcal{P}_{\alpha}=\bigoplus_{j \in J} k_{i} W
$$

for some $J \subset\{1, \ldots, \ell\}$ and hence $\mathcal{P}_{\alpha}$ is equivalent to $\sum_{i \in J} \rho_{k_{i}}$ as a $K_{\circ}$ module.

\footnotetext{
${ }^{2}$ We can write $M=\mathbb{Z}_{2} \ltimes U(V)$ where $\mathbb{Z}_{2}$ acts on $H_{V}$ via $f$ and on $U(V)$ via $k \mapsto \bar{k}$.
} 
Since there are at most $\ell$ factors in Equation 2.4, at least $N 2^{\ell}$ distinct $\mathcal{P}_{\alpha}$ 's must contain copies of $\rho$. As there are only $2^{\ell}$ possibilities for $J$, at least $N$ of these $\mathcal{P}_{\alpha}$ 's must be equivalent as $K_{\circ}$-modules.

We have shown that for each $N$, one can find $N$ distinct irreducible $K$ modules that are equivalent as $K_{\circ}$-modules. This is impossible since $K / K_{\circ}$ is a finite group.

As explained in Section 1, Proposition 2.2 allows us to restrict our attention to connected subgroups of $U(V)$. Henceforth, unless otherwise stated, $K$ will always denote a compact connected subgroup of $U(V)$.

2.2. Invariants and orbits. The following result is well known. A proof can be found on page 133 in [28].

Proposition 2.3. Let $K$ be a compact group acting smoothly on a real vector space $W$. Then the invariants $\mathbb{C}[W]^{K}$ separate $K$-orbits in $W$.

Suppose now that $K \subset U(V)$ where $V$ is a complex vector space. Proposition 2.3 shows that $\mathbb{C}\left[V_{\mathbb{R}}\right]^{K}$ separates $K$-orbits in $V$. If $V \cong \mathbb{C}^{n}$ then $\mathbb{C}[V] \cong \mathbb{C}\left[z_{1}, \ldots, z_{n}\right]$ and $\mathbb{C}\left[V_{\mathbb{R}}\right] \cong \mathbb{C}\left[z_{1}, \ldots, z_{n}, \bar{z}_{1}, \ldots, \bar{z}_{n}\right]$. Note that the holomorphic $K$-invariants $\mathbb{C}[V]^{K}$ need not separate $K$-orbits. In particular, when $\left(K, H_{V}\right)$ is a Gelfand pair, $1_{K}$ occurs in $\mathbb{C}[V]$ with multiplicity 1 and it follows that the only $K$-invariant holomorphic polynomials on $V$ are constants.

Other applications of Proposition 2.3 in this paper concern the adjoint action of $K$ on $\mathfrak{k}$ and the coadjoint action of $K$ on $\mathfrak{k}^{*}$.

Corollary 2.4. Let $K$ be a compact connected Lie group with Lie algebra $\mathfrak{k}$. The $\operatorname{Ad}(K)$-invariant polynomials $I[\mathfrak{k}]:=\mathbb{C}[\mathfrak{k}]^{\operatorname{Ad}(K)}$ separate the adjoint orbits in $\mathfrak{k}$. Similarly, $I\left[\mathfrak{k}^{*}\right]:=\mathbb{C}\left[\mathfrak{k}^{*}\right]^{\operatorname{Ad}^{*}(K)}$ separates the coadjoint orbits in $\mathfrak{k}^{*}$.

2.3. The orbit method for compact groups. We recall how the orbit method for a compact group $K$ is related to the classical theory of highest weights. For details, we refer the reader to [12] or [24]. Let $T$ be a maximal torus in $K$ with Lie algebra $\mathfrak{t} \subset \mathfrak{k}$ and let $\langle\cdot \mid \cdot\rangle$ be a negative definite $A d(K)$ invariant inner product on $\mathfrak{k}$. We identify $\mathfrak{t}^{*}$ with the subset $\{\langle X \mid \cdot\rangle \mid X \in \mathfrak{t}\}$ of $\mathfrak{k}^{*}$. Each coadjoint orbit in $\mathfrak{k}^{*}$ meets $\mathfrak{t}^{*} \subset \mathfrak{k}^{*}$ in an orbit of the Weyl group. A point $\alpha \in \mathfrak{t}^{*}$ is an integral point provided

$$
X \in \operatorname{Ker}(\exp : \mathfrak{t} \rightarrow T) \text { implies that } \alpha(X) \in \mathbb{Z} \text {. }
$$


In this case, if $i \alpha: \mathfrak{t} \rightarrow i \mathbb{R}$ is the highest weight for some representation $\eta_{\alpha}$, then $\mathcal{O}_{\alpha}^{K}$ corresponds to $\eta_{\alpha}$ via the orbit method. For $K \subset U(n)$, a suitable pairing $\langle\cdot \mid \cdot\rangle$ as above is given by

$$
\langle A \mid B\rangle:=\operatorname{tr}(A B)
$$

Remark 2.1. Although $K$ denotes a subgroup of $U(V)$, in some of the examples $K$ merely acts on $V$ by some unitary representation. In this case one can replace $K$ by its image in $U(V)$ but we need to be careful when speaking of integral points if the representation is not faithful. In this setting, an integral point $\alpha \in \mathfrak{t}^{*}$ is one for which

$$
X \in \operatorname{Ker}(\mathfrak{t} \underset{\exp }{\longrightarrow} T \rightarrow U(V)) \text { implies that } \alpha(X) \in \mathbb{Z} .
$$

2.4. Spectrum of the quasi-regular representation. We supply here a result which we believe must be known but which we could not locate in the literature.

Proposition 2.5 Let $G$ be type $I$ and $K \subset G$ a compact subgroup. Then $\pi \in \widehat{G}$ is weakly contained in the quasi-regular representation $\operatorname{Ind}_{K}^{G}\left(1_{K}\right)$ if and only if $\pi$ is weakly contained in the regular representation and contains a $K$-fixed vector.

Corollary 2.6. If $G$ is amenable and type I (in particular, for $G=K \ltimes H_{V}$ ), $\pi$ is weakly contained in the quasi-regular representation if and only if $\pi$ contains a $K$-fixed vector.

The corollary is an immediate consequence of Proposition 2.5 since every irreducible unitary representation of an amenable group is weakly contained in its regular representation.

Proof of Proposition 2.5. Since $K$ is compact, the quasi-regular representation is a subrepresentation of the regular representation. Thus any representation weakly contained in the quasi-regular representation is also weakly contained in the regular representation. Furthermore, it follows from the Ahn Reciprocity Theorem (see [23, pp.56-58]) that a.e. $\pi \in \widehat{G}$ contains a $K$-fixed vector. Since this is a closed condition in the Fell topology, the "only if" portion of the proposition is clear.

Conversely, let $\pi$ be in the reduced dual and let $\xi$ be a $K$-fixed vector in the space of $\pi$. The matrix coefficient $\langle\pi(g) \xi, \xi\rangle$ is uniformly approximable on compacta by matrix coefficients of the regular representation, i.e. by functions of the form $h_{1} * h_{2}$ where $h_{1}, h_{2} \in L^{2}(G)$. Let $\mathcal{U} \subset G$ be compact 
and assume (without loss of generality) that $K \mathcal{U} K=\mathcal{U}$. Averaging the $h_{j}$ over $K$ does not disturb an approximation to the matrix coefficient on $\mathcal{U}$. Hence, the approximation can be made using $K$-invariant functions. Since $\pi$ is irreducible, this implies that $\pi$ is weakly contained in $\operatorname{Ind}_{K}^{G}\left(1_{K}\right)$.

In light of Corollary 2.6, the spectrum of $\operatorname{Ind}_{K}^{G}\left(1_{K}\right)$ is exactly the set

$$
\widehat{G}_{K}:=\{\pi \in \widehat{G} \mid \pi \text { contains a } K \text {-fixed vector }\} .
$$

Alternatively, there is no ambiguity in referring to the members of the set $\widehat{G}_{K}$ as the irreducibles which "occur $i n$ " $\operatorname{Ind}_{K}^{G}\left(1_{K}\right)$.

2.5. Coadjoint orbits in $G=K \ltimes H_{V}$. We identify the Lie algebra $\mathfrak{h}_{V}$ of $H_{V}$ with $H_{V}$ via the exponential map. The Lie bracket on $\mathfrak{h}_{V}$ is given by

$$
\left[(z, t),\left(z^{\prime}, t^{\prime}\right)\right]=\left(0,-\omega\left(z, z^{\prime}\right)\right)
$$

and the derived action of the Lie algebra $\mathfrak{k} \subset \mathfrak{u}(V) \cong \mathfrak{u}(n)$ of $K \subset U(V)$ on $\mathfrak{h}_{V}$ is

$$
A \cdot(z, t)=(A z, 0) .
$$

Points in $\mathfrak{g}=\mathfrak{k} \ltimes \mathfrak{h}_{V}$ will be written as triples $(A, z, t)$ where $A \in \mathfrak{k}$ and $(z, t) \in \mathfrak{h}_{V}$. Using the Baker-Campbell-Hausdorff formula, one sees that

$$
\operatorname{Exp}_{G}\left(s A_{1}, s z_{1}, s t_{1}\right)=\left(\operatorname{Exp}_{K}\left(s A_{1}\right), s z_{1}+O\left(s^{2}\right), s t_{1}\right) .
$$

Thus, we compute

$$
\begin{aligned}
& \operatorname{Ad}(k, z, t)\left(A_{1}, z_{1}, t_{1}\right)=\left.\frac{d}{d s}\right|_{0}\left[(k, z, t) \operatorname{Exp}_{G}\left(s A_{1}, s z_{1}, s t_{1}\right)(k, z, t)^{-1}\right] \\
&=\left.\frac{d}{d s}\right|_{0}\left[(k, z, t)\left(\operatorname{Exp}_{K}\left(s A_{1}\right), s z_{1}, s t_{1}\right)\left(k^{-1},-k^{-1} z,-t\right)\right] \\
&=\left.\frac{d}{d s}\right|_{0}\left[\left(k \operatorname{Exp}_{K}\left(s A_{1}\right), z+s k z_{1}, t+s t_{1}-\frac{s}{2} \omega\left(z, k z_{1}\right)\right)\left(k^{-1},-k^{-1} z,-t\right)\right] \\
&=\left.\frac{d}{d s}\right|_{0}\left(k \operatorname{Exp}_{K}\left(s A_{1}\right) k^{-1}, z+s k z_{1}-k \operatorname{Exp}_{K}\left(s A_{1}\right) k^{-1} z, s t_{1}\right. \\
&\left.-\frac{s}{2} \omega\left(z, k z_{1}\right)+\frac{1}{2} \omega\left(z, k \operatorname{Exp}_{K}\left(s A_{1}\right) k^{-1} z\right)+\frac{s}{2} \omega\left(k z_{1}, k \operatorname{Exp}_{K}\left(s A_{1}\right) k^{-1} z\right)\right) \\
&=\left.\left(\operatorname{Ad}_{K}(k) A_{1}, k z_{1}-\left(\operatorname{Ad}_{K}(k) A_{1}\right) z, t_{1}+\omega\left(k z_{1}, z\right)+\frac{1}{2} \omega\left(z, \operatorname{Ad}_{K}(k) A_{1}\right) z\right)\right) .
\end{aligned}
$$

That is,

$$
\operatorname{Ad}(k, z, t)\left(A_{1}, z_{1}, t_{1}\right)
$$




$$
=\left(k \cdot A_{1}, k z_{1}-\left(k \cdot A_{1}\right) z, t_{1}+\omega\left(k z_{1}, z\right)+\frac{1}{2} \omega\left(z,\left(k \cdot A_{1}\right) z\right)\right)
$$

where we now write $k \cdot A$ for the adjoint action of $K$ on $\mathfrak{k}$. Note in particular that

$$
\operatorname{Ad}(k)\left(A_{1}, z_{1}, t_{1}\right)=\left(k \cdot A_{1}, k z_{1}, t_{1}\right) .
$$

For $z \in V$, define $z^{*} \in V^{*}$ by

$$
z^{*}\left(z^{\prime}\right):=\omega\left(z, z^{\prime}\right)
$$

We define a map $\times: V \times V \rightarrow \mathfrak{k}^{*},\left(z, z^{\prime}\right) \mapsto z \times z^{\prime}$ by

$$
z \times z^{\prime}(A):=\left(z^{\prime}\right)^{*}(A z)=\omega\left(z^{\prime}, A z\right) \text { for } A \in \mathfrak{k} .
$$

It is easy to verify that for $k \in K, A \in \mathfrak{k}$ and $z, z^{\prime} \in V$ one has

$$
\begin{aligned}
k z^{*} & :=z^{*} \circ k^{-1}=(k z)^{*} \\
z^{*} \circ A & =-(A z)^{*} \\
z \times z^{\prime} & =z^{\prime} \times z \\
k \cdot\left(z \times z^{\prime}\right) & =(k z) \times\left(k z^{\prime}\right) .
\end{aligned}
$$

We will identify $\mathfrak{g}^{*}=\left(\mathfrak{k} \ltimes \mathfrak{h}_{V}\right)^{*}=\mathfrak{h}_{V}^{\perp} \oplus \mathfrak{k}^{\perp} \cong \mathfrak{k}^{*} \oplus \mathfrak{h}_{V}^{*}$ with $\mathfrak{k}^{*} \oplus \mathfrak{h}_{V}$. Specifically, we will write $\mu_{(\alpha, z, \lambda)}$ to denote the element in $\mathfrak{g}^{*}$ given by

$$
\mu_{(\alpha, z, \lambda)}\left(A, z^{\prime}, t\right):=\alpha(A)+z^{*}\left(z^{\prime}\right)+\lambda t .
$$

For $\alpha \in \mathfrak{k}^{*}, \mu \in \mathfrak{g}^{*}$ and $k \in K$ we write $k \cdot \alpha$ and $k \cdot \mu$ as shorthand for $\operatorname{Ad}^{*}(k)(\alpha)$ and $A d^{*}(k)(\mu)$. In view of Equations 2.13 and 2.16, we have

$$
k \cdot \mu_{(\alpha, z, \lambda)}=\mu_{(k \cdot \alpha, k z, t)}
$$

Working from Equations 2.12, 2.16 and 2.19 we compute

$$
\begin{aligned}
\operatorname{Ad}^{*}\left((k, z, t)^{-1}\right) \mu_{\left(\alpha, z_{\circ}, \lambda\right)}\left(A_{1}, z_{1}, t_{1}\right) \\
=\mu_{\left(\alpha, z_{\circ}, \lambda\right)}\left(\operatorname{Ad}(k, z, t)\left(A_{1}, z_{1}, t_{1}\right)\right) \\
=\alpha\left(k \cdot A_{1}\right)+z_{\circ}^{*}\left(k z_{1}\right)-z_{\circ}^{*}\left(\left(k \cdot A_{1}\right) z\right)+\lambda t_{1} \\
\quad+\lambda \omega\left(k z_{1}, z\right)+\frac{\lambda}{2} \omega\left(z,\left(k \cdot A_{1}\right) z\right) \\
=\left(k^{-1} \cdot \alpha-k^{-1} \cdot\left(z \times z_{\circ}\right)+\frac{\lambda}{2} k^{-1} \cdot(z \times z)\right)\left(A_{1}\right) \\
\quad+\left(\left(k^{-1} z_{\circ}\right)^{*}-\lambda\left(k^{-1} z\right)^{*}\right)\left(z_{1}\right)+\lambda t_{1} .
\end{aligned}
$$


Replacing $(k, z, t)$ above by $(k, z, t)^{-1}=\left(k^{-1},-k^{-1} z,-t\right)$ yields the following formula for the coadjoint action of $G$ on $\mathfrak{g}^{*}$.

Lemma 2.7 $\operatorname{Ad}^{*}(k, z, t) \mu_{\left(\alpha, z_{\circ}, \lambda\right)}=\mu_{\left(k \cdot \alpha+z \times k z_{\circ}+\frac{\lambda}{2} z \times z, k z_{\circ}+\lambda z, \lambda\right)}$ where $z \times z^{\prime}(A):=$ $\left(z^{\prime}\right)^{*}(A z)=\omega\left(z^{\prime}, A z\right)$.

Letting $k$ and $z$ vary over $K$ and $V$ respectively, we see that the coadjoint orbit $\mathcal{O}_{\left(\alpha, z_{o}, \lambda\right)}^{G}$ through $\mu_{\left(\alpha, z_{\circ}, \lambda\right)}$ is

$$
\mathcal{O}_{\left(\alpha, z_{\circ}, \lambda\right)}^{G}=\left\{\mu_{\left(k \cdot \alpha+z \times k z_{\circ}+\frac{\lambda}{2} z \times z, k z_{\circ}+\lambda z, \lambda\right)} \mid k \in K, z \in V\right\}
$$

or equivalently, replacing $z$ by $k z$ and using Equation 2.21,

$$
\mathcal{O}_{\left(\alpha, z_{\circ}, \lambda\right)}^{G}=\left\{k \cdot \mu_{\left(\alpha+z \times z_{\circ}+\frac{\lambda}{2} z \times z, z_{\circ}+\lambda z, \lambda\right)} \mid k \in K, z \in V\right\} .
$$

\section{Reformulations of conditions ( $\mathrm{OC}$ ) and (SC).}

In this section we will derive versions of Conditions (OC) and (SC) that involve only the actions of $K$ on $V$ and $\mathbb{C}[V]$. A key ingredient is an unnormalized version of the moment map for a Lie group representation [29].

Definition 3.1. Define the moment map $\tau: V \rightarrow \mathfrak{k}^{*}$ for the action of $K$ on $V$ by $\tau(z):=z \times z$ for $z \in V$. That is, $\tau(z)(A)=\omega(z, A z)$.

Note that since $\langle z, A z\rangle$ is pure imaginary, one can also write $\tau(z)(A)=$ $\frac{1}{i}\langle z, A z\rangle=i\langle A z, z\rangle$. The moment map satisfies

$$
\begin{aligned}
\tau(c z)=|c|^{2} \tau(z) & \text { for } c \in \mathbb{C}, \\
\tau(k z)=k \cdot \tau(z) & \text { for } k \in K .
\end{aligned}
$$

We will also consider the induced algebra map,

$$
\tau^{*}: \mathbb{C}\left[\mathfrak{k}^{*}\right] \rightarrow \mathbb{C}\left[V_{\mathbb{R}}\right], \quad\left(\tau^{*}(p)\right)(v):=p(\tau(v)) .
$$

In view of Equation $3.2, \tau^{*}$ is $K$-equivariant and yields an algebra map

$$
\tau^{*}: I\left[\mathfrak{k}^{*}\right] \rightarrow \mathbb{C}\left[V_{\mathbb{R}}\right]^{K}
$$

by restriction to the $\operatorname{Ad}^{*}(K)$-invariant polynomials.

If we identify $V$ with $\mathbb{C}^{n}$ by using some orthonormal basis for $V$, then $K$ can be regarded as a subgroup of the $n$ by $n$ unitary matrices $U(n)$, and $\mathfrak{k}$ as a subalgebra of the skew Hermitian matrices $\mathfrak{u}(n)$. An easy computation shows that $\tau$ is given by the formula

$$
\tau(z)(A)=i \sum_{j, \ell} a_{j, \ell} z_{\ell} \overline{z_{j}}=i \operatorname{tr}\left(A z z^{*}\right), \quad z \in V, A \in \mathfrak{k} .
$$


Here we regard $z$ as a column vector $z=\left[\begin{array}{c}z_{1} \\ \vdots \\ z_{n}\end{array}\right]$ and $z^{*}:=\bar{z}^{t}$. So $i z z^{*}$ is the $n$ by $n$ skew Hermitian matrix whose entries are given by $\left(i z z^{*}\right)_{\ell, j}=i z_{\ell} \overline{z_{j}}$.

The algebra map $\tau^{*}: \mathbb{C}\left[\mathfrak{k}^{*}\right] \rightarrow \mathbb{C}\left[V_{\mathbb{R}}\right]$ is determined by its behavior on homogeneous polynomials of degree 1 . For $A \in \mathfrak{k}$, let

$$
\psi_{A} \in \mathfrak{k}^{* *} \subset \mathbb{C}\left[\mathfrak{k}^{*}\right] \quad \text { be given by } \psi_{A}(\alpha)=\alpha(A) .
$$

Then

$$
\tau^{*}\left(\psi_{A}\right)(z)=i \operatorname{tr}\left(A z z^{*}\right)
$$

Remark 3.1. If one uses a negative definite $\operatorname{Ad}(K)$-invariant inner product on $\mathfrak{k}$ to identify $\mathfrak{k}$ with $\mathfrak{k}^{*}$, then $\tau$ and $\tau^{*}$ become maps $\tau: V \rightarrow \mathfrak{k}$ and $\tau^{*}: I[\mathfrak{k}] \rightarrow \mathbb{C}\left[V_{\mathbb{R}}\right]^{K}$. Such identifications are useful when working with examples. In particular, for the action of $U(n)$ on $\mathbb{C}^{n}$ one has $\tau(z)=i z z^{*} \in \mathfrak{u}(n)$ when one uses the pairing 2.6 to identify $\mathfrak{u}(n)^{*}$ with $\mathfrak{u}(n)$.

Our first goal is to reformulate Condition (OC). We must consider intersections of the form $\mathcal{O}_{\left(\alpha, z_{o}, \lambda\right)}^{G} \cap \mathfrak{k}^{\perp}$. We will write points in $\mathfrak{h}_{V}^{*}$ as $\nu_{(z, \lambda)}$, where $\nu_{(z, \lambda)}\left(z^{\prime}, t\right):=z^{*}\left(z^{\prime}\right)+\lambda t$ and identify $\mathfrak{k}^{\perp}$ with $\mathfrak{h}_{V}^{*}$ via $\mu_{(0, z, \lambda)} \leftrightarrow \nu_{(z, \lambda)}$. Note that this identification is $\operatorname{Ad}^{*}(G)$-equivariant. Equation 2.23 shows that

$$
\begin{aligned}
& \mathcal{O}_{\left(\alpha, z_{\circ}, \lambda\right)}^{G} \cap \mathfrak{k}^{\perp} \\
& =\left\{k \cdot \nu_{\left(z_{\circ}+\lambda z, \lambda\right)} \mid k \in K \text { and } z \in V \text { with } \alpha+z \times z_{\circ}+\frac{\lambda}{2} \tau(z)=0\right\} .
\end{aligned}
$$

In particular, $\mathcal{O}_{\left(\alpha, z_{0}, \lambda\right)}^{G} \cap \mathfrak{k}^{\perp}$ is $K$-saturated.

We shall refer to the orbits $\mathcal{O}_{\left(\alpha, z_{0}, \lambda\right)}^{G}$ with $\lambda \neq 0$ as the generic orbits in $\mathfrak{g}^{*}$. In this case, Equation 2.23 shows that $\mathcal{O}_{\left(\alpha, z_{\circ}, \lambda\right)}^{G}$ contains the point $\mu_{\left(\alpha-\frac{1}{2 \lambda} \tau\left(z_{\circ}\right), 0, \lambda\right)}$. Thus, we may as well take $z_{\circ}=0$ here. Note that the orbits $\mathcal{O}_{(\alpha, 0, \lambda)}^{G}$ are all distinct, so that $\left(\mathfrak{k}^{*} / K\right) \times(\mathbb{R} \backslash\{0\})$ parameterizes the generic coadjoint orbits in $\mathfrak{g}^{*}$. We have

$$
\mathcal{O}_{(\alpha, 0, \lambda)}^{G} \cap \mathfrak{k}^{\perp}=\left\{k \cdot \nu_{(z, \lambda)} \mid \tau(z)=-2 \lambda \alpha\right\}=\left\{\nu_{(z, \lambda)} \mid \tau(z) \in \mathcal{O}_{-2 \lambda \alpha}^{K}\right\} .
$$

Fixing $\lambda=\lambda_{\circ} \neq 0$ and letting $\alpha=-\frac{1}{2 \lambda_{\circ}} \beta$ vary over $\mathfrak{k}^{*}$, we see that Condition (OC) holds for all orbits $\mathcal{O}_{\left(\alpha, 0, \lambda_{\circ}\right)}^{G}$ if and only if $\tau^{-1}\left(\mathcal{O}_{\beta}^{K}\right)$ consists of at most one $K$-orbit in $V$. As this condition is independent of $\lambda_{\circ}$, (OC) holds for 
all generic orbits if and only if $\tau$ induces an injective map $V / K \rightarrow \mathfrak{k}^{*} / K$ on $K$-orbits.

Non-generic coadjoint orbits have the form

$$
\mathcal{O}_{\left(\alpha, z_{\circ}, 0\right)}^{G}=\left\{k \cdot \mu_{\left(\alpha+z \times z_{\circ}, z_{\circ}, 0\right)} \mid k \in K, z \in V\right\} .
$$

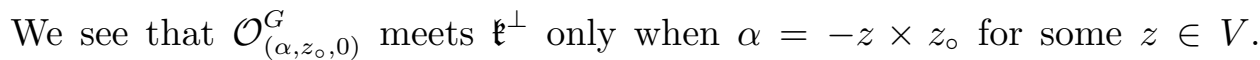
In this case, $\mathcal{O}_{\left(\alpha, z_{\circ}, 0\right)}^{G} \cap \mathfrak{k}^{\perp}=\left\{\nu_{\left(k z_{0}, 0\right)} \mid k \in K\right\}$ is a single $K$-orbit. That is, Condition (OC) always holds for the non-generic orbits.

Theorem 3.1. The following conditions are equivalent for $K \subset U(V)$ :

(1) $\left(K, H_{V}\right)$ satisfies Condition (OC).

(2) The moment map $\tau: V \rightarrow \mathfrak{k}^{*}$ is injective on $K$-orbits. That is, if $\mathcal{O}_{\tau(z)}^{K}=\mathcal{O}_{\tau\left(z^{\prime}\right)}^{K}$ then $K z=K z^{\prime}$.

(3) If $z \times z^{\prime}=0$ then $K\left(z+z^{\prime}\right)=K\left(z-z^{\prime}\right)$.

(4) The algebra $\tau^{*}\left(I\left[\mathfrak{k}^{*}\right]\right) \subset \mathbb{C}\left[V_{\mathbb{R}}\right]^{K}$ separates $K$-orbits in $V$.

Proof. We have seen that Condition (OC) holds for all coadjoint orbits $\mathcal{O}_{\left(\alpha, z_{o}, \lambda\right)}^{G}$ if and only if (OC) holds for the generic orbits $\mathcal{O}_{(\alpha, 0, \lambda)}^{G}$. As explained above, this is equivalent to $\tau$ being injective on $K$-orbits. To see that (2) and (3) are equivalent, note that

$$
\tau\left(z \pm z^{\prime}\right)=\tau(z)+\tau\left(z^{\prime}\right) \pm 2 z \times z^{\prime} .
$$

Here we have used Equation 2.18.

Suppose that $K z \neq K z^{\prime}$. If $(2)$ holds then $\mathcal{O}_{\tau(z)}^{K} \neq \mathcal{O}_{\tau\left(z^{\prime}\right)}^{K}$. As $I\left[\mathfrak{k}^{*}\right]$ separates $K$-coadjoint orbits (Corollary 2.4), there is a polynomial $p \in I\left[\mathfrak{k}^{*}\right]$ with $p\left(\mathcal{O}_{\tau(z)}^{K}\right) \neq p\left(\mathcal{O}_{\tau\left(z^{\prime}\right)}^{K}\right)$. But then $\tau^{*}(p)(K z) \neq \tau^{*}(p)\left(K z^{\prime}\right)$. This shows that (2) implies (4). On the other hand, if (4) holds and $K z \neq K z^{\prime}$ then for some $p \in I\left[\mathfrak{k}^{*}\right]$ we have $\tau^{*}(p)(K z) \neq \tau^{*}(p)\left(K z^{\prime}\right)$. Thus $p\left(\mathcal{O}_{\tau(z)}^{K}\right) \neq$ $p\left(\mathcal{O}_{\tau\left(z^{\prime}\right)}^{K}\right)$ and we have $\mathcal{O}_{\tau(z)}^{K} \neq \mathcal{O}_{\tau\left(z^{\prime}\right)}^{K}$. Hence, (4) implies (2).

Next, we will reformulate Condition (SC) in terms of the moment map and the representation of $K$ on $\mathbb{C}[V] . \eta_{\alpha}$ will denote the representation of $K$ corresponding to an integral coadjoint orbit $\mathcal{O}_{\alpha}^{K} \subset \mathfrak{k}^{*}$.

Theorem 3.2. $\left(K, H_{V}\right)$ satisfies Condition (SC) if and only if the spectrum of the representation of $K$ on $\mathbb{C}[V]$ is

$$
\left\{\eta_{\alpha} \mid \alpha \text { is an integral point in } \tau(V) \subset \mathfrak{k}^{*}\right\} \text {. }
$$


Proof. First consider an integral non-generic coadjoint orbit $\mathcal{O}_{\left(\alpha, z_{\circ}, 0\right)}^{G}$. The corresponding representation $\rho_{\left(\alpha, z_{\circ}\right)}$ of $G$ is the identity on $Z\left(H_{V}\right) \subset G$ and factors through the group $K \ltimes V$. This may be described via the Mackey machine as follows. (See [24] for details on this.) Let $\chi_{z_{\circ}}$ be the unitary character on $H_{V}$ defined by

$$
\chi_{z_{\circ}}(z, t):=e^{i\left(z_{\circ}, z\right)} \quad \text { where as before }(\cdot, \cdot):=\operatorname{Re}\langle\cdot, \cdot\rangle .
$$

The stabilizer of $\chi_{z_{\circ}}$ in $K$ is

$$
K_{z_{\circ}}=\left\{k \in K \mid \chi_{z_{\circ}} \circ k=\chi_{z_{\circ}}\right\}=\left\{k \in K \mid k z_{\circ}=z_{\circ}\right\} .
$$

Let $\mathfrak{k}_{z_{\circ}}$ be the Lie algebra of $K_{z_{\circ}}$ and $\alpha_{\circ}:=\left.\alpha\right|_{\mathfrak{k}_{z_{\circ}}} \in \mathfrak{k}_{z_{\circ}}^{*}$. Integrality of $\mathcal{O}_{\left(\alpha, z_{\circ}, 0\right)}^{G}$ is equivalent to the integrality of $\alpha_{\circ}$ for the group $K_{z_{\circ}}$. Let $\gamma_{\alpha}$ be the unitary representation of $K_{z_{\circ}}$ which corresponds to the coadjoint orbit $\mathcal{O}_{\alpha_{\circ}}^{K_{z_{\circ}}}$. The representation $\rho_{\left(\alpha, z_{\circ}\right)}$ of $G$ given by $\mathcal{O}_{\left(\alpha, z_{\circ}, 0\right)}^{G}$ is

$$
\rho_{\left(\alpha, z_{\circ}\right)}=\operatorname{Ind}_{K_{z_{\circ}} \ltimes H_{V}}^{K \ltimes H_{V}}\left(\gamma_{\alpha} \otimes \chi_{z_{\circ}}\right)
$$

and hence

$$
\left.\rho_{\left(\alpha, z_{\circ}\right)}\right|_{K}=\operatorname{Ind}_{K_{z_{0}}}^{K}\left(\gamma_{\alpha}\right) .
$$

Thus $1_{K}$ occurs in $\left.\rho_{\left(\alpha, z_{\circ}\right)}\right|_{K}$ if and only if $\gamma_{\alpha}$ occurs in $\left.1_{K}\right|_{K_{z_{\circ}}}=1_{K_{z_{\circ}}}$. This can happen only when $\gamma_{\alpha}=1_{K_{z_{0}}}$. Since $1_{K_{z_{0}}}$ corresponds to the coadjoint orbit $\{0\}$ in $\mathfrak{k}_{z_{\circ}}^{*}$, we see that $1_{K}$ occurs in $\left.\rho_{\left(\alpha, z_{\circ}\right)}\right|_{K}$ if and only if $\alpha_{\circ}=0$.

Equation 3.10 shows that $\mathcal{O}_{\left(\alpha, z_{\circ}, 0\right)}^{G}$ meets $\mathfrak{k}^{\perp}$ when $\alpha=z \times z_{\circ}$ for some $z \in$ $V$. For $A \in \mathfrak{k}_{z_{\circ}}=\left\{A \in \mathfrak{k} \mid A z_{\circ}=0\right\}$, one has $\left(z \times z_{\circ}\right)(A)=\left(z_{\circ} \times z\right)(A)=$ $\omega\left(z, A z_{\circ}\right)=0$. Thus if $\mathcal{O}_{\left(\alpha, z_{\circ}, 0\right)}^{G}$ is an integral orbit that meets $\mathfrak{k}^{\perp}$, then $1_{K}$ occurs in $\left.\rho_{\left(\alpha, z_{\circ}\right)}\right|_{K}$. On the other hand, consider the linear map

$$
\beta: V \rightarrow\left(\mathfrak{k} / \mathfrak{k}_{z_{\circ}}\right)^{*}, \quad \beta(z):=(i z) \times z_{\circ} .
$$

One sees easily that $\operatorname{Ker}(\beta)=\left\{z \mid\left(z, A z_{\circ}\right)=0\right.$ for all $\left.A \in \mathfrak{k}\right\}=\left(\mathfrak{k} z_{\circ}\right)^{\perp}$. Hence, $\operatorname{dim}(\operatorname{Ker}(\beta))=\operatorname{dim}(V)-\operatorname{dim}\left(\mathfrak{k} z_{\circ}\right)$ and $\operatorname{dim}(\beta(V))=\operatorname{dim}\left(\mathfrak{k} z_{\circ}\right)=$ $\operatorname{dim}\left(K z_{\circ}\right)=\operatorname{dim}\left(K / K_{z_{\circ}}\right)=\operatorname{dim}\left(\left(\mathfrak{k} / \mathfrak{k}_{z_{\circ}}\right)^{*}\right)$. That is, $\beta$ is onto and hence if $\left.\alpha\right|_{\mathfrak{k}_{z_{\circ}}}=0$, one must have that $\alpha=z \times z_{\circ}$ for some $z \in V$. Thus if $1_{K}$ occurs in $\left.\rho_{\left(\alpha, z_{o}\right)}\right|_{K}$, then $\mathcal{O}_{\left(\alpha, z_{\circ}, 0\right)}^{G}$ meets $\mathfrak{k}^{\perp}$. In other words, we have shown that for non-generic integral coadjoint orbits $\mathcal{O}_{\xi} \subset \mathfrak{g}^{*}, \rho_{\xi}$ occurs in $\operatorname{Ind}_{K}^{G}\left(1_{K}\right)$ if and only if $\mathcal{O}_{\xi}$ meets $\mathfrak{k}^{\perp}$.

Next consider the generic orbits $\mathcal{O}_{(\alpha, 0, \lambda)}^{G}$. Such an orbit is integral if and only if $\mathcal{O}_{\alpha}^{K}$ is integral in $\mathfrak{k}^{*}$. Write $\eta_{\alpha}$ for the corresponding representation 
of $K$ acting in say $\mathcal{H}_{\alpha}$. Let $\pi_{\lambda} \in \widehat{H_{V}}$ acting in say $\mathcal{F}_{\lambda}$ have central character $\pi_{\lambda}(0, t)=e^{i \lambda t}$. It is well known that $\pi_{\lambda}$ is determined up to unitary equivalence by its central character. Since $K$ preserves $\mathcal{O}_{\nu_{(0, \lambda)}}^{H_{V}}, \pi_{\lambda} \circ k$ is unitarily equivalent to $\pi_{\lambda}$ for each $k \in K$. That is, the stabilizer of $\pi_{\lambda}$ is all of $K$. Let $W_{\lambda}(k)$ intertwine $\pi_{\lambda}$ and $\pi_{\lambda} \circ k$,

$$
\pi_{\lambda}(k z, t)=W_{\lambda}(k) \pi_{\lambda}(z, t) W_{\lambda}(k)^{-1} .
$$

We will see below that we can choose a $W_{\lambda}(k)$ for each $k \in K$ in such a way that $W_{\lambda}$ is a unitary representation of $K$ on $\mathcal{F}_{\lambda}$. The representation $\rho_{(\alpha, \lambda)}$ of $G$ corresponding to $\mathcal{O}_{(\alpha, 0, \lambda)}^{G}$ can be realized in $\mathcal{H}_{\alpha} \otimes \mathcal{F}_{\lambda}$ as

$$
\rho_{(\alpha, \lambda)}(k, z, t)=\eta_{\alpha}(k) \otimes\left[\pi_{\lambda}(z, t) \circ W_{\lambda}(k)\right] .
$$

(See [24] for details.) In particular, $\rho_{(\alpha, \lambda)}(k)=\eta_{\alpha}(k) \otimes W_{\lambda}(k)$. We conclude that $1_{K}$ occurs in $\left.\rho_{(\alpha, \lambda)}\right|_{K}$ if and only if $\overline{\eta_{\alpha}}=\eta_{-\alpha}$ occurs in $W_{\lambda}$.

Suppose that $\lambda>0$. As explained in [2], we can realize $\pi_{\lambda}$ in a Fock space $\mathcal{F}_{\lambda}$ consisting of entire functions $V \rightarrow \mathbb{C}$ that are square integrable with respect to the measure $\left(\frac{\lambda}{2 \pi}\right)^{n} e^{-\frac{\lambda}{2}|z|^{2}} d z d \bar{z}$ on $V$. $W_{\lambda}$ is given by $\left(W_{\lambda}(k) f\right)(z)=$ $f\left(k^{-1} z\right)$. The holomorphic polynomials $\mathbb{C}[V]$ are dense in $\mathcal{F}_{\lambda}$ and $W_{\lambda}(K)$ preserves $\mathbb{C}[V]$. Thus $1_{K}$ occurs in $\left.\rho_{(\alpha, \lambda)}\right|_{K}$ if and only if $\eta_{-\alpha}$ occurs in $\mathbb{C}[V]$. On the other hand, $\mathcal{O}_{(\alpha, 0, \lambda)}^{G}$ meets $\mathfrak{k}^{\perp}$ when $-2 \lambda \alpha \in \tau(V)$. Equivalently, in view of Equation 3.1, we must have $-\alpha \in \tau(V)$. Thus Condition (SC) holds for the integral orbits $\mathcal{O}_{(\alpha, 0, \lambda)}^{G}$ with $\lambda>0$ if and only if for all integral $\alpha \in \mathfrak{k}^{*}$, $\eta_{\alpha}$ occurs in $\mathbb{C}[V]$ exactly when $\alpha$ belongs to the image of $\tau$.

Next suppose that $\lambda<0$. In this case, $\pi_{\lambda}=\overline{\pi_{|\lambda|}}$ can be realized in $\overline{\mathcal{F}_{|\lambda|}}$. The intertwining representation becomes $W_{\lambda}=\overline{W_{|\lambda|}}$, so that $\rho_{(\alpha, \lambda)}(k)=$ $\eta_{\alpha}(k) \otimes \overline{W_{|\lambda|}(k)}$. Thus, $1_{K}$ occurs in $\rho_{(\alpha, \lambda)}$ if and only if $\eta_{\alpha}$ occurs in $W_{|\lambda|}$. Equivalently, $\eta_{\alpha}$ must occur in $\mathbb{C}[V]$. On the other hand, $\mathcal{O}_{(\alpha, 0, \lambda)}^{G}$ will meet $\mathfrak{k}^{\perp}$ when $-2 \lambda \alpha=2|\lambda| \alpha$ belongs to $\tau(V)$. As for the case $\lambda>0$, Condition (SC) holds for the integral orbits $\mathcal{O}_{(\alpha, 0, \lambda)}^{G}$ with $\lambda<0$ if and only if the spectrum of $\mathbb{C}[V]$ is given by the integral points $\alpha \in \tau(V)$.

Example 3.1. The prototypical Gelfand pair is of course $\left(U(n), H_{n}\right)$. We will show that Conditions (OC) and (SC) hold for this example. Identifying $\mathfrak{u}(n)^{*}$ with $\mathfrak{u}(n)$ via the $U(n)$-invariant negative definite bilinear form $\langle A \mid B\rangle:=$ $\operatorname{tr}(A B)$ (see Section 2.3), Equation 3.5 shows that $\tau: V \rightarrow \mathfrak{u}(n)$ is given by $\tau(z)=i z z^{*}$. This is a skew-Hermitian matrix with eigenvalues $i|z|^{2}$ of multiplicity 1 and 0 of multiplicity $n-1$. Thus, if $\tau(z)$ is conjugate to $\tau\left(z^{\prime}\right)$ via a unitary matrix then $|z|=\left|z^{\prime}\right|$ and hence, $z$ and $z^{\prime}$ belong to a common $U(n)$-orbit. This shows that Condition (OC) holds for $\left(U(n), H_{n}\right)$. 
The standard maximal torus $T$ in $U(n)$ consists of the diagonal matrices

$$
k_{\theta}:=\left[\begin{array}{lll}
e^{i \theta_{1}} & & \\
& \ddots & \\
& & e^{i \theta_{n}}
\end{array}\right]
$$

with Lie algebra $\mathfrak{t}=\left\{A_{\theta}\right\}$ where

$$
A_{\theta}:=\left[\begin{array}{ccc}
i \theta_{1} & & \\
& \ddots & \\
& & i \theta_{n}
\end{array}\right] .
$$

The multiplicity free decomposition of $\mathbb{C}\left[\mathbb{C}^{n}\right]$ is just

$$
\mathbb{C}\left[\mathbb{C}^{n}\right]=\sum_{m=0}^{\infty} \mathcal{P}_{m}
$$

where $\mathcal{P}_{m}$ is the space of homogeneous polynomials of degree $m$. Writing $p_{J}(z):=z_{1}^{j_{1}} \cdots z_{n}^{j_{n}}$, one has $k_{\theta} \cdot p_{J}=e^{-i J \cdot \theta} p_{J}$. Thus, $p_{m}(z):=p_{(m, 0, \cdots, 0)}(z)=$ $z_{1}^{m}$ is a highest weight vector in $\mathcal{P}_{m}$ with highest weight $-i m \epsilon_{1}$ where $\epsilon_{j}\left(A_{\theta}\right):=\theta_{j}$. Our computation of $\tau: \mathbb{C}^{n} \rightarrow \mathfrak{u}(n)^{*}$ shows that $\tau(U(n) z)$ meets $\mathfrak{t}^{*}=\left\{\left\langle A_{\theta} \mid \cdot\right\rangle\right\} \subset \mathfrak{u}(n)^{*}$ in the Weyl orbit

$$
\left\{-|z|^{2} \epsilon_{1}, \ldots,-|z|^{2} \epsilon_{n}\right\} \text {. }
$$

We see that the integral orbits in $\tau(V)$ are exactly those through $\alpha_{m}:=-m \epsilon_{1}$ for $m=0,1, \ldots$ as desired.

\section{The spectrum of $\operatorname{Ind}_{K}^{G}\left(1_{K}\right)$.}

The following theorem shows that a weakened version of Condition (SC) is always true.

Proposition 4.1. If $\rho_{\xi}$ belongs to the spectrum $\widehat{G}_{K}$ of $\operatorname{Ind}_{K}^{G}\left(1_{K}\right)$ then $\mathcal{O}_{\xi}^{G} \cap$ $\mathfrak{k}^{\perp} \neq \phi$. Equivalently, if $\eta_{\alpha}$ occurs in the representation of $K$ on $\mathbb{C}[V]$ then $\mathcal{O}_{\alpha}^{K} \subset \tau(V)$.

Proof. The argument given in the proof of Theorem 3.2 shows that $\widehat{G}_{K} \subset\left\{\rho_{\xi} \mid \xi \in \mathfrak{k}^{\perp}\right.$ is integral $\}$ if and only if

$$
\operatorname{Spec}_{K}(\mathbb{C}[V]) \subset\left\{\eta_{\alpha} \mid \alpha \text { is an integral point in } \tau(V) \subset \mathfrak{k}^{*}\right\} .
$$

Thus, the two assertions in Proposition 4.1 are equivalent. 
Suppose that $\eta_{\alpha}$ occurs in the space of homogeneous polynomials $\mathcal{P}_{m}$ of degree $m$ in $\mathbb{C}[V]$. Let $\mathcal{O}_{m}^{U(V)} \subset \mathfrak{u}(V)^{*}$ be the coadjoint orbit corresponding to the representation $\left(\widetilde{\eta_{m}}\right.$ say) of $U(V)$ on $\mathcal{P}_{m}$. (In terms of the notation used in Example 3.1, $\mathcal{O}_{m}^{U(V)}=U(V) \cdot \alpha_{m}$.) Since $\eta_{\alpha}$ occurs in $\left.\widetilde{\eta_{m}}\right|_{K}$ it follows from a result of Heckman [12] that $\mathcal{O}_{\alpha}^{K} \subset \pi\left(\mathcal{O}_{m}^{U(V)}\right)$ where $\pi: \mathfrak{u}(V)^{*} \rightarrow \mathfrak{k}^{*}$ denotes the restriction map. Example 3.1 shows that $\mathcal{O}_{m}^{U(V)}$ is in the image of the moment map $\tau_{U(V)}: V \rightarrow \mathfrak{u}(V)^{*}$. These observations prove Proposition 4.1 since $\tau_{K}: V \rightarrow \mathfrak{k}^{*}$ is related to $\tau_{U(V)}: V \rightarrow \mathfrak{u}(V)^{*}$ by $\tau_{K}=\pi \circ \tau_{U(V)}$.

Proposition 4.1 is also proved in [22], but from an "un-reformulated" perspective - i.e. without using the moment map.

Example 4.1. We can have $\widehat{G}_{K} \varsubsetneqq\left\{\rho_{\xi} \mid \xi \in \mathfrak{k}^{\perp}\right.$ is integral $\}$. That is, one can have $\mathcal{O}_{\xi}^{G} \cap \mathfrak{k}^{\perp} \neq \phi$ for some integral orbit $\mathcal{O}_{\xi}^{G}$, even though $\rho_{\xi}$ is not in the spectrum of $\operatorname{Ind}_{K}^{G}\left(1_{K}\right)$. Consider the action of $K:=\mathbb{T}$ (the unit circle in $\mathbb{C})$ on $V:=\mathbb{C}^{2}$ given by $k \cdot\left(z_{1}, z_{2}\right):=\left(k^{2} z_{1}, k^{3} z_{2}\right)$. For $p_{(j, \ell)}(z):=z_{1}^{j} z_{2}^{\ell}$ one has $k \cdot p_{(j, \ell)}=k^{-(2 j+3 \ell)} p_{(j, \ell)}$. The Lie algebra of $K$ is $\mathfrak{t}=i \mathbb{R}$. The character $k \mapsto k^{m}$ for $m \in \mathbb{Z}$ corresponds to the one-point coadjoint orbit $\mathcal{O}_{m}:=$ $\{i a \mapsto m a\}$. Thus, the coadjoint orbits that correspond to representations of $K$ occurring in $\mathbb{C}[V]$ are

$$
\left\{\mathcal{O}_{-(2 j+3 \ell)} \mid j, \ell \geq 0\right\}=\left\{\mathcal{O}_{0}, \mathcal{O}_{-2}, \mathcal{O}_{-3}, \ldots\right\}
$$

On the other hand, $\tau: V \rightarrow \mathfrak{k}^{*}$ is given by

$$
\tau(z)(i a)=-a\left(2\left|z_{1}\right|^{2}+3\left|z_{2}\right|^{2}\right)
$$

and the integral orbits in the image of $\tau$ are $\left\{\mathcal{O}_{0}, \mathcal{O}_{-1}, \mathcal{O}_{-2}, \ldots\right\}$. In particular, $\mathcal{O}_{-1}=\tau\left(K \cdot\left(\frac{1}{\sqrt{2}}, 0\right)\right)$ is in the image of $\tau$ but the corresponding representation of $K$ does not appear in $\mathbb{C}[V]$.

Note that $\mathbb{C}[V]$ is not $K$-multiplicity free, so $\left(K, H_{V}\right)$ is not a Gelfand pair. Moreover, Condition (OC) fails here since, for example, $\mathcal{O}_{-1}$ is also the image of $K \cdot\left(0, \frac{1}{\sqrt{3}}\right)$. It is harder to find examples of Gelfand pairs where Condition (SC) fails. One such example is 7.2 presented below.

\section{Geometric criteria.}

In this section, we will prove Theorem 1.3. This will also establish Theorem 1.2 since the two results are equivalent in view of Theorem 3.1. Our proof uses some results from [15] and [18] concerning the derived action of the Lie algebra of $K$ on the space $\mathbb{C}[V]$. We begin by relating this action to the moment map. 
Let $\mathcal{P} \mathcal{D}(V)$ be the algebra of differential operators with (holomorphic) polynomial coefficients on $V$. We have $\mathcal{P} \mathcal{D}(V) \hookrightarrow$ End $(\mathbb{C}[V])$, that is each $D \in \mathcal{P} \mathcal{D}(V)$ acts on $\mathbb{C}[V] . K_{c}$ acts on $\mathcal{P} \mathcal{D}(V)$ by conjugation inside End $(\mathbb{C}[V])$,

$$
((g \cdot D)(p))(z)=(D(p \circ g))\left(g^{-1} z\right), \quad D \in \mathcal{P} \mathcal{D}(V), g \in K_{c}, p \in \mathbb{C}[V] .
$$

The derived action of $\mathfrak{k}_{c}$ on $\mathbb{C}[V]$ is given by

$$
(X \cdot p)(z):=\left.\frac{d}{d s}\right|_{0} p(\exp (-s X) z)
$$

The map $d \iota: \mathfrak{k}_{c} \rightarrow \mathcal{P} \mathcal{D}(V)$ given by $(d \iota)(X) p:=X \cdot p$ extends to a $K_{c^{-}}$ equivariant algebra map

$$
d \iota: \mathcal{U}\left(\mathfrak{k}_{c}\right) \rightarrow \mathcal{P} \mathcal{D}(V)
$$

where $\mathcal{U}\left(\mathfrak{k}_{c}\right)=\mathcal{U}(\mathfrak{k}) \otimes \mathbb{C}$ is the complexified enveloping algebra of $\mathfrak{k}$ and $K_{c}$ acts on $\mathcal{U}\left(\mathfrak{k}_{c}\right)$ via Ad. Restricting to $K_{c}$-invariant elements yields a map

$$
d \iota: \mathcal{Z U}\left(\mathfrak{k}_{c}\right) \rightarrow \mathcal{P D}(V)^{K_{c}}
$$

where $\mathcal{Z U}\left(\mathfrak{k}_{c}\right)$ denotes the center of $\mathcal{U}\left(\mathfrak{k}_{c}\right)$.

Proposition 5.1. There are vector space isomorphisms $\lambda: I\left[\mathfrak{k}^{*}\right] \rightarrow \mathcal{Z U}\left(\mathfrak{k}_{c}\right)$ and $\Gamma: \mathbb{C}\left[V_{\mathbb{R}}\right]^{K} \rightarrow \mathcal{P} \mathcal{D}(V)^{K_{c}}$ for which the diagram

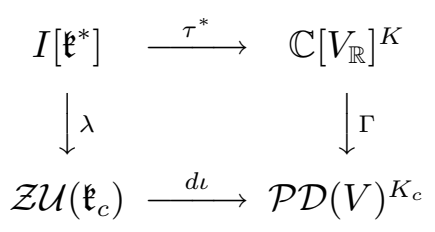

commutes.

Proof. Suppose that $\mathcal{B}=\left\{e_{1}, \ldots, e_{n}\right\}$ is an $\langle\cdot, \cdot\rangle$-orthonormal basis for $V$ and let $\left(z_{1}, \ldots, z_{n}\right)$ denote coordinates with respect to $\mathcal{B}$. We use this to identify $V$ with $\mathbb{C}^{n}$ and $K$ with a subgroup of the $n$ by $n$ unitary matrices $U(n)$. $\mathfrak{k}$ becomes a subalgebra of the skew Hermitian matrices $\mathfrak{u}(n)$. For $A=\left[a_{j, \ell}\right] \in \mathfrak{k}$, one computes

$$
(A \cdot p)(z)=\left.\frac{d}{d s}\right|_{0} p\left(z-s A z+O\left(s^{2}\right)\right)=-\sum_{j, \ell} a_{j, \ell} z_{\ell} \frac{\partial p}{\partial z_{j}} .
$$


That is, $d \iota$ can be written in coordinates as

$$
d \iota(A)=-\sum_{j, \ell} a_{j, \ell} z_{\ell} \frac{\partial}{\partial z_{j}} \quad \text { for } A \in \mathfrak{k} \subset \mathcal{U}\left(\mathfrak{k}_{c}\right) .
$$

We will relate $d \iota$ to $\tau^{*}$.

For $v \in V$, let $\epsilon_{v}:=\langle\cdot, v\rangle \in V^{*} \subset \mathbb{C}[V]$. Thus also $\overline{\epsilon_{v}}=\overline{\langle\cdot, v\rangle}=\langle v, \cdot\rangle \in$ $(\bar{V})^{*} \subset \mathbb{C}[\bar{V}]$. Note that $\left\{\epsilon_{v} \mid v \in V\right\}$ and $\left\{\bar{\epsilon}_{v} \mid v \in V\right\}$ generate the algebras $\mathbb{C}[V]$ and $\mathbb{C}[\bar{V}]$ respectively. The algebra isomorphism $\mathbb{C}[V] \otimes \mathbb{C}[\bar{V}] \cong \mathbb{C}\left[V_{\mathbb{R}}\right]$ given by $(p \otimes q) \leftrightarrow(z \mapsto p(z) q(z))$ is $K$-equivariant where $K$ acts on each of $\mathbb{C}\left[V_{\mathbb{R}}\right], \mathbb{C}[V]$ and $\mathbb{C}[\bar{V}]$ by the formula $(k \cdot f)(z):=f\left(k^{-1} z\right)$. Note that $k \cdot \epsilon_{v}=\epsilon_{k v}$ and $k \cdot \overline{\epsilon_{v}}=\overline{\epsilon_{k v}}$.

Define an algebra map

$$
M: \mathbb{C}[V] \rightarrow \mathcal{P} \mathcal{D}(V)
$$

by polynomial multiplication, $M(p) q:=p q$. We have

$$
(k \cdot M(p)) q(z)=M(p)(q \circ k)\left(k^{-1} z\right)=p\left(k^{-1} z\right) q(z)=(M(k \cdot p) q)(z) .
$$

Thus, $M$ is $K$-equivariant. For $v \in V$, let $\partial_{v} \in \mathcal{P} \mathcal{D}(V)$ be

$$
\left(\partial_{v} p\right)(z):=\left.\frac{d}{d s}\right|_{0} p(z+s v) .
$$

Since the $\partial_{v}$ 's commute in $\mathcal{P} \mathcal{D}(V)$, we obtain an algebra map

$$
\Delta: \mathbb{C}[\bar{V}] \rightarrow \mathcal{P D}(V)
$$

given by $\Delta\left(\overline{\epsilon_{v}}\right)=\partial_{v}$ on the generators. This is also $K$-equivariant. Indeed,

$$
\begin{aligned}
\Delta\left(k \cdot \overline{\epsilon_{v}}\right)(p)(z)=\Delta\left(\overline{\epsilon_{k v}}\right) p(z) & =\left.\frac{d}{d s}\right|_{0} p(z+s k v) \\
& =\left.\frac{d}{d s}\right|_{0}(p \circ k)\left(k^{-1} z+s v\right) \\
& =\partial_{v}(p \circ k)\left(k^{-1} z\right) \\
& =\left(k \cdot \partial_{v}\right)(p)(z)=\left(k \cdot \Delta\left(\overline{e_{v}}\right)\right)(p)(z) .
\end{aligned}
$$

Let $\Gamma: \mathbb{C}\left[V_{\mathbb{R}}\right] \rightarrow \mathcal{P} \mathcal{D}(V)$ be given by

$$
\Gamma: \mathbb{C}\left[V_{\mathbb{R}}\right] \cong \mathbb{C}[V] \otimes \mathbb{C}[\bar{V}] \underset{M \otimes \Delta}{\longrightarrow} \mathcal{P} \mathcal{D}(V) .
$$

$\Gamma$ is a vector space isomorphism and is $K$-equivariant since both $M$ and $\Delta$ are $K$-equivariant. It is not, however, an algebra map because $M(\mathbb{C}[V])$ 
and $\Delta(\mathbb{C}[\bar{V}])$ do not commute in $\mathcal{P} \mathcal{D}(V)$. Writing $\epsilon_{j}$ and $\overline{\epsilon_{j}}$ for $\epsilon_{e_{j}}$ and $\overline{\epsilon_{e_{j}}}$, Equation 3.7 becomes

$$
\tau^{*}\left(\psi_{A}\right)=i \sum_{j, \ell} a_{j, \ell} \epsilon_{\ell} \otimes \overline{\epsilon_{j}}
$$

As $\epsilon_{j}(z)=z_{j}$ and $\overline{\epsilon_{\ell}}(z)=\overline{z_{\ell}}$ this yields

$$
\Gamma\left(\tau^{*}\left(\psi_{A}\right)\right)=i \sum_{j, \ell} a_{j, \ell} z_{\ell} \frac{\partial}{\partial z_{j}} .
$$

The map

$$
\lambda: \mathfrak{k}^{* *} \rightarrow \mathcal{U}\left(\mathfrak{k}_{c}\right), \quad \psi_{A} \mapsto-i A
$$

extends to a variant of the usual symmetrization map [13]

$$
\lambda: \mathbb{C}\left[\mathfrak{k}^{*}\right] \rightarrow \mathcal{U}\left(\mathfrak{k}_{c}\right)
$$

characterized by $\lambda\left(\psi_{A}^{m}\right)=(-i)^{m} A^{m}$. This is a $K$-equivariant vector space isomorphism but not an algebra map.

The maps introduced above fit together to form the diagram:

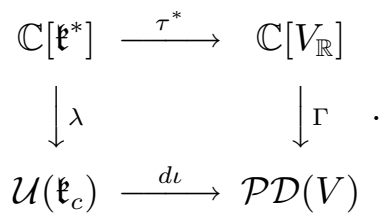

The diagram commutes in view of Equations 5.7 and 5.13. The horizontal maps are algebra maps and the vertical maps are vector space isomorphisms but not algebra maps. All maps are $K$-equivariant. Restricting to $K$-invariants yields the commutative diagram (5.5) in the statement of Proposition 5.1.

As noted in the above proof, $\lambda$ and $\Gamma$ are not algebra maps. However, one does have, for example, that for $p, q \in \mathbb{C}\left[V_{\mathbb{R}}\right]^{K}$,

$$
\Gamma(p) \Gamma(q)=\Gamma(p q)+\Delta(p, q)
$$

where $\Delta(p, q)$ is an operator of lower total degree than $\Gamma(p q)$. We use this fact together with Proposition 5.1 to prove the following lemma.

Lemma 5.2. $\mathbb{C}\left[V_{\mathbb{R}}\right]^{K}$ is a finitely generated module over $\tau^{*}\left(I\left[\mathfrak{k}^{*}\right]\right)$ if and only if $\mathcal{P} \mathcal{D}(V)^{K_{c}}$ is finitely generated as a module over $d_{\iota}\left(\mathcal{Z U}\left(\mathfrak{k}_{c}\right)\right)$. 
Proof. Suppose that $p_{1}, p_{2}, \ldots, p_{r} \in \mathbb{C}\left[V_{\mathbb{R}}\right]^{K}$ generate $\mathbb{C}\left[V_{\mathbb{R}}\right]^{K}$ as a $\tau^{*}\left(I\left[\mathfrak{k}^{*}\right]\right)$ module and let $D_{j}:=\Gamma\left(p_{j}\right)$. We can assume that $p_{1}=1$, so the scalar operators in $\mathcal{P} \mathcal{D}(V)^{K_{c}}$ are multiples of $D_{1}=I$. Assume inductively that elements in $\mathcal{P} \mathcal{D}(V)^{K_{c}}$ with degree less that $m$ can be written in the form $A_{1} D_{1}+\cdots+A_{r} D_{r}$ for some $A_{1}, \ldots, A_{r} \in d \iota\left(\mathcal{Z U}\left(\mathfrak{k}_{c}\right)\right)$. Let $D \in \mathcal{P} \mathcal{D}(V)^{K_{c}}$ have degree $m$ and write $\Gamma^{-1}(D)=a_{1} p_{1}+\cdots+a_{r} p_{r}$ for some $a_{1}, \ldots, a_{r} \in \tau^{*}\left(I\left[\mathfrak{k}^{*}\right]\right)$. Thus

$$
\begin{aligned}
D & =\Gamma\left(a_{1} p_{1}\right)+\Gamma\left(a_{2} p_{2}\right)+\cdots+\Gamma\left(a_{r} p_{r}\right) \\
& =A_{1} D_{1}+A_{2} D_{2}+\cdots+A_{r} D_{r}+\Delta
\end{aligned}
$$

where $A_{j}:=\Gamma\left(a_{j}\right) \in \operatorname{d}\left(\mathcal{Z U}\left(\mathfrak{k}_{c}\right)\right)$ and $\operatorname{deg}(\Delta)<m$. By the inductive hypothesis, we can write $\Delta=B_{1} D_{1}+B_{2} D_{2}+\cdots B_{r} D_{r}$ for some $B_{1}, \ldots, B_{r} \in$ $d \iota\left(\mathcal{Z U}\left(\mathfrak{k}_{c}\right)\right)$. Thus

$$
D=C_{1} D_{1}+C_{2} D_{2}+\cdots+C_{r} D_{r}
$$

where $C_{j}:=A_{j}+B_{j} \in d \iota\left(\mathcal{Z U}\left(\mathfrak{k}_{c}\right)\right)$. This shows that $D_{1}, D_{2}, \ldots, D_{r}$ generate $\mathcal{P D}(V)^{K_{c}}$ as a $d \iota\left(\mathcal{Z U}\left(\mathfrak{k}_{c}\right)\right)$-module.

Next suppose that $D_{1}, D_{2}, \ldots, D_{r}$ generate $\mathcal{P} \mathcal{D}(V)^{K_{c}}$ as a module over $d \iota\left(\mathcal{Z U}\left(\mathfrak{k}_{c}\right)\right)$ and let $p_{j}:=\Gamma^{-1}\left(D_{j}\right)$. We can assume that $D_{1}$ is the identity operator. The constants in $\mathbb{C}\left[V_{\mathbb{R}}\right]^{K}$ are thus multiples of $p_{1}=1$. Assume inductively that if $\operatorname{deg}(\Gamma(q))<m$ then $q \in \mathbb{C}\left[V_{\mathbb{R}}\right]^{K}$ can be written as $q=$ $a_{1} p_{1}+a_{2} p_{2}+\cdots a_{r} p_{r}$ for some $a_{1}, \ldots, a_{r} \in \tau^{*}\left(I\left[\mathfrak{k}^{*}\right]\right)$. Let $p \in \mathbb{C}\left[V_{\mathbb{R}}\right]^{K}$ be such that $\operatorname{deg}(\Gamma(p))=m$ and write $\Gamma(p)=A_{1} D_{1}+A_{2} D_{2}+\cdots+A_{r} D_{r}$ for some $A_{1}, \ldots, A_{r} \in d \iota\left(\mathcal{Z U}\left(\mathfrak{k}_{c}\right)\right)$. Thus, $\Gamma(p)=\Gamma\left(a_{1} p_{1}+a_{2} p_{2}+\cdots a_{r} p_{r}\right)+\Delta$ where $a_{j}=\Gamma^{-1}\left(A_{j}\right) \in \tau^{*}\left(I\left[\mathfrak{k}^{*}\right]\right)$ and $\operatorname{deg}(\Delta)<m$. By the inductive hypothesis, $\Delta=\Gamma\left(b_{1} p_{1}+b_{2} p_{2}+\cdots b_{r} p_{r}\right)$ for some $b_{1}, \ldots, b_{r} \in \tau^{*}\left(I\left[\mathfrak{k}^{*}\right]\right)$. Thus, $p=$ $c_{1} p_{1}+c_{2} p_{2}+\cdots+c_{r} p_{r}$ where $c_{j}:=a_{j}+b_{j} \in \tau^{*}\left(I\left[\mathfrak{k}^{*}\right]\right)$. This shows that $p_{1}, p_{2}, \ldots, p_{r}$ generate $\mathbb{C}\left[V_{\mathbb{R}}\right]^{K}$ as a $\tau^{*}\left(I\left[\mathfrak{k}^{*}\right]\right)$-module.

The proof of Theorem 1.3 will be carried out in two stages, represented by Propositions 5.3 and 5.4 below. These assert that the multiplicity free condition and injectivity of the moment map on $K$-orbits are each equivalent to the same algebraic condition concerning the ring extension $\tau^{*}\left(I\left[\mathfrak{k}^{*}\right]\right) \subset$ $\mathbb{C}\left[V_{\mathbb{R}}\right]^{K}$. We write $\operatorname{Frac}(R)$ for the fraction field of an integral domain $R$.

Proposition 5.3. The action of $K$ on $V$ is multiplicity free if and only if $\operatorname{Frac}\left(\mathbb{C}\left[V_{\mathbb{R}}\right]^{K}\right)$ is a finite extension of the field $\operatorname{Frac}\left(\tau^{*}\left(I\left[\mathfrak{k}^{*}\right]\right)\right)$.

Proposition 5.4. $\tau$ is one-to-one on $K$-orbits if and only if $\operatorname{Frac}\left(\mathbb{C}\left[V_{\mathbb{R}}\right]^{K}\right)$ is a finite extension of the field $\operatorname{Frac}\left(\tau^{*}\left(I\left[\mathfrak{k}^{*}\right]\right)\right)$. 
Proof of Proposition 5.3. Suppose that the action of $K$ on $V$ is multiplicity free. Thus $\mathcal{P D}(V)^{K_{c}}$ is abelian by (7) in Theorem 1.1. It follows from the Main Theorem part (a) in $\left[\mathbf{1 8}\right.$, p. 254] that $\mathcal{P} \mathcal{D}(V)^{K_{c}}$ is finitely generated as a module over $d \iota\left(\mathcal{Z U}\left(\mathfrak{k}_{c}\right)\right)$ and hence $\mathbb{C}\left[V_{\mathbb{R}}\right]^{K}$ is a finitely generated $\tau^{*}\left(I\left[\mathfrak{k}^{*}\right]\right)$-module by Lemma 5.2. Thus $\operatorname{Frac}\left(\mathbb{C}\left[V_{\mathbb{R}}\right]^{K}\right)$ is a finite extension of $\operatorname{Frac}\left(\tau^{*}\left(I\left[\mathfrak{k}^{*}\right]\right)\right)$.

Let $\mathcal{Z}:=\mathcal{Z}\left(\mathcal{P D}(V)^{K_{c}}\right)$ denote the center of $\mathcal{P} \mathcal{D}(V)^{K_{c}}$ and let $\mathcal{A}:=$ $\Gamma^{-1}(\mathcal{Z})$. It follows from Diagram 5.5 that $\mathcal{A} \supseteq \tau^{*}\left(I\left[\mathfrak{k}^{*}\right]\right)$. Theorems 9.4 and 9.5 in $[\mathbf{1 8}]$ show that one always has:

(1) $\mathcal{A}$ is a subring of $\mathbb{C}\left[V_{\mathbb{R}}\right]^{K}$.

(2) $\mathcal{P} \mathcal{D}(V)^{K_{c}}$ is a free $\mathcal{Z}$-module.

(3) $\mathbb{C}\left[V_{\mathbb{R}}\right]^{K}$ is a free $\mathcal{A}$-module.

Suppose that $\operatorname{Frac}\left(\mathbb{C}\left[V_{\mathbb{R}}\right]^{K}\right)$ is a finite extension of $\operatorname{Frac}\left(\tau^{*}\left(I\left[\mathfrak{k}^{*}\right]\right)\right)$. Thus, $\operatorname{Frac}\left(\mathbb{C}\left[V_{\mathbb{R}}\right]^{K}\right)$ is also a finite extension of $\operatorname{Frac}(\mathcal{A})$. Let $N:=\left[\operatorname{Frac}\left(\mathbb{C}\left[V_{\mathbb{R}}\right]^{K}\right)\right.$ : $\operatorname{Frac}(\mathcal{A})]<\infty$. It follows that an $\mathcal{A}$-module base for $\mathbb{C}\left[V_{\mathbb{R}}\right]^{K}$ can contain no more than $N$ elements. Indeed, one sees easily that if $p_{1}, p_{2}, \ldots, p_{M} \in$ $\mathbb{C}\left[V_{\mathbb{R}}\right]^{K}$ and $M>N$ then one can find $a_{1}, a_{2}, \ldots, a_{M} \in \mathcal{A}$ not all zero with $a_{1} p_{1}+a_{2} p_{2}+\cdots+a_{M} p_{M}=0$. Thus, $\mathbb{C}\left[V_{\mathbb{R}}\right]^{K}$ is finitely generated as an $\mathcal{A}$-module. As in Lemma 5.2, it follows using Diagram (5.5) and induction that $\mathcal{P} \mathcal{D}(V)^{K_{c}}$ is a finitely generated free $\mathcal{Z}$-module.

Let $D_{1}, D_{2}, \ldots, D_{\ell}$ be a $\mathcal{Z}$-module base for $\mathcal{P} D(V)^{K_{c}}$. Then, a typical element $D \in \mathcal{P} \mathcal{D}(V)^{K_{c}}$ can be written as $D=A_{1} D_{1}+\cdots A_{\ell} D_{\ell}=D_{1} A_{1}+$ $\cdots D_{\ell} A_{\ell}$ for some $A_{1}, \ldots, A_{\ell} \in \mathcal{Z}$.

Let $\mathcal{J}_{\sigma}$ be the $K$-isotypic component in $\mathbb{C}[V]$ for $\sigma \in \widehat{K}$. Choose a maximal torus in $K$ and let $\mathcal{H}_{\sigma}$ be the subspace of highest weight vectors in $\mathcal{J}_{\sigma}$. Note that $\operatorname{dim}\left(\mathcal{H}_{\sigma}\right)$ is the multiplicity of $\sigma$ in $\mathbb{C}[V]$. The proof of Proposition 7.1 in [15] shows that $\mathcal{P} \mathcal{D}(V)^{K_{c}}$ acts irreducibly on $\mathcal{H}_{\sigma}$. Thus for $p \in \mathcal{H}_{\sigma}, p \neq 0$, we have $\mathcal{H}_{\sigma}=\mathcal{P} \mathcal{D}(V)^{K_{c}} p$.

Let $D=D_{1} A_{1}+\cdots+D_{\ell} A_{\ell} \in \mathcal{P} \mathcal{D}(V)^{K_{c}}$ as above. Since $A_{j}$ commutes with the action of $\mathcal{P} \mathcal{D}(V)^{K_{c}}$, it acts on $\mathcal{H}_{\sigma}$ by some scalar, $\lambda_{j}$ say. Thus, $D p=\lambda_{1} D_{1} p+\cdots+\lambda_{\ell} D_{\ell} p$. We conclude that $\mathcal{H}_{\sigma} \subset \operatorname{Span}\left\{D_{1} p, \ldots, D_{\ell} p\right\}$. In particular, $\operatorname{dim}\left(\mathcal{H}_{\sigma}\right) \leq \ell$. This shows that the multiplicity of any representation $\sigma \in \widehat{K}$ in $\mathbb{C}[V]$ is uniformly bounded by $\ell$. Lemma 2.1 now implies that the action of $K$ on $V$ is multiplicity free.

Proof of Proposition 5.4. Assume that $\operatorname{Frac}\left(\mathbb{C}\left[V_{\mathbb{R}}\right]^{K}\right)$ is a finite extension of $\operatorname{Frac}\left(\tau^{*}\left(I\left[\mathfrak{k}^{*}\right]\right)\right)$ and let $\gamma_{1}, \ldots, \gamma_{\ell}$ be algebra generators for $\mathbb{C}\left[V_{\mathbb{R}}\right]^{K}$. Thus each $\gamma_{j}$ is algebraic over $\operatorname{Frac}\left(\tau^{*}\left(I\left[\mathfrak{k}^{*}\right]\right)\right)$. Suppose that

$$
a_{0, j}+a_{1, j} \gamma_{j}+a_{2, j} \gamma_{j}^{2}+\cdots+a_{d_{j}, j} \gamma_{j}^{d_{j}}=0
$$

where $a_{i, j}=\tau^{*}\left(c_{i, j}\right)$ and $c_{i, j} \in I\left[\mathfrak{k}^{*}\right]$. 
Let $v_{\circ} \in V$ and $v \in \tau^{-1}\left(\tau\left(K v_{\circ}\right)\right)$. Evaluating Equation 5.17 at $v$ shows that $\gamma_{j}(v)$ is a root of the polynomial

$$
c_{0, j}\left(\tau\left(v_{\circ}\right)\right)+c_{1, j}\left(\tau\left(v_{\circ}\right)\right) X+\cdots+c_{d_{j}, j}\left(\tau\left(v_{\circ}\right)\right) X^{d_{j}} .
$$

Thus $\gamma:=\gamma_{1} \times \gamma_{2} \times \cdots \times \gamma_{\ell}: V \rightarrow \mathbb{C}^{\ell}$ assumes at most $M:=d_{1} d_{2} \cdots d_{\ell}$ distinct values on $\tau^{-1}\left(\tau\left(K v_{\circ}\right)\right)$. Since the level sets of $\gamma$ are the $K$-orbits in $V$ (by Proposition 2.3), we conclude that $\tau^{-1}\left(\tau\left(K v_{\circ}\right)\right.$ ) is finite union of $K$-orbits. One the other hand, Theorem 4.6 in [21] asserts that $\tau^{-1}\left(\tau\left(K v_{\circ}\right)\right)$ is always a connected subset of $V$. Hence $\tau^{-1}\left(\tau\left(K v_{\circ}\right)\right)=K v_{\circ}$, so $\tau$ is oneto-one on $K$-orbits.

Conversely, suppose that $\tau$ is one-to-one on $K$-orbits. Let $q_{1}, q_{2}, \ldots, q_{r}$ be $\mathbb{R}$-valued algebra generators for $\tau^{*}\left(I\left[\mathfrak{k}^{*}\right]\right)$ and let

$$
L_{v, j}:=\left\{w \in V \mid q_{\ell}(w)=q_{\ell}(v) \quad \text { for } \ell=1, \ldots, j\right\}
$$

for $v \in V$ and $j=1, \ldots, r$. Note that $L_{v, r}=\tau^{-1}(\tau(K v))=K v$ since $\tau$ is one-to-one on $K$-orbits.

Suppose that $q_{j+1}$ depends algebraically on $q_{1}, \ldots, q_{j}$. That is, suppose that

$$
\sum c_{\ell}\left(q_{1}, \ldots, q_{j}\right) q_{j+1}^{\ell}=0
$$

for some polynomials $c_{\ell}\left(q_{1}, \ldots, q_{j}\right)$ in $q_{1}, \ldots, q_{j}$. Evaluating at $w \in L_{v, j}$, shows that $q_{j+1}(w)$ is a root of $\sum c_{\ell}\left(q_{1}(v), \ldots, q_{j}(v)\right) X^{\ell}$. Thus, $q_{j+1}$ assumes only a finite number of values on $L_{v, j}$. Hence $q_{j+1}$ is constant on the connected components of $L_{v, j}$. After perhaps rearranging the $q_{j}$ 's we see that one can find some index $m \leq r$ for which $q_{1}, \ldots, q_{m}$ are algebraically independent (over $\mathbb{R}$ ) and $q_{m+1}, \ldots, q_{r}$ are constant on the connected components of the level sets $L_{v, m}$. In particular, the connected component of $v$ in $L_{v, m}$ coincides with the connected component of $v$ in $L_{v, r}$, which is $K v$ by hypothesis.

Consider the derivative $D\left(q_{1} \times \cdots \times q_{m}\right)$ of $q_{1} \times \cdots \times q_{m}: V \rightarrow \mathbb{R}^{m}$. Let

$$
m^{\prime}:=\max \left\{\operatorname{rank}\left(D\left(q_{1} \times \cdots \times q_{m}\right)(v)\right) \mid v \in V\right\} .
$$

We will see below that in fact $m^{\prime}=m$. Let $v_{\circ} \in V$ be a generic point. That is, $\operatorname{rank}\left(D\left(q_{1} \times \cdots \times q_{m}\right)\left(v_{\circ}\right)\right)=m^{\prime}$ and $m^{\prime}$ is the codimension of the level set $L_{v_{\circ}, m}$, which coincides with $K v_{\circ}$ in a neighborhood of $v_{\circ}$. By reordering $q_{1}, \ldots, q_{m}$, we can assume that $\operatorname{rank}\left(D\left(q_{1} \times \cdots \times q_{m^{\prime}}\right)\left(v_{\circ}\right)\right)=m^{\prime}$ and hence that $D\left(q_{1} \times \cdots \times q_{m^{\prime}}\right)$ has rank $m^{\prime}$ at all points in some open neighborhood $U$ of $v_{\circ}$. Thus, the $K$-orbits meeting $U$ form a foliation of $U$ with codimension $m^{\prime}$. 
Let $A$ denote the orthogonal complement (with respect to $(\cdot, \cdot))$ in $V_{\mathbb{R}}$ of the tangent space to $K v_{\circ}$ at $v_{\circ} . A$ is an affine subspace of $V_{\mathbb{R}}$. Choose (real) coordinates on $V_{\mathbb{R}}$ so that $A=\left\{\left(x, a_{\circ}\right) \mid x \in \mathbb{R}^{m^{\prime}}\right\}$ and $v_{\circ}=\left(0, a_{\circ}\right)$. By shrinking $U$, we can assume that $A$ is a cross section to the $K$-orbits through $U$. (That is, $K v \cap A \neq \emptyset$ for all $v \in U$.)

Let $\widetilde{q}_{j}$ denote the polynomial function on $\mathbb{R}^{m^{\prime}}$ defined by $\widetilde{q}_{j}(x):=q_{j}\left(x, a_{\circ}\right)$. It follows that $\widetilde{q}_{1}, \ldots, \widetilde{q}_{m^{\prime}}$ are algebraically independent over $\mathbb{R}$. Indeed, if we had some polynomial relation $p\left(\widetilde{q}_{1}, \ldots, \widetilde{q}_{m^{\prime}}\right)=0$ then $p\left(q_{1}, \ldots, q_{m^{\prime}}\right)$ would vanish on $A$. Since the $K$-orbit through any point in $U$ meets $A$, $p\left(q_{1}, \ldots, q_{m^{\prime}}\right)$ vanishes on $U$ by $K$-invariance. Since $p\left(q_{1}, \ldots, q_{m^{\prime}}\right)$ is a polynomial function on $V_{\mathbb{R}}$, it follows that $p\left(q_{1}, \ldots, q_{m^{\prime}}\right)$ is identically zero on $V_{\mathbb{R}}$. This contradicts the algebraic independence of $q_{1}, \ldots, q_{m^{\prime}}$.

Finally, consider the field inclusion $\mathbb{R}\left(\widetilde{q}_{1}, \ldots, \widetilde{q}_{m^{\prime}}\right) \hookrightarrow \mathbb{R}\left(x_{1}, \ldots, x_{m^{\prime}}\right)$. Since both fields have transcendence degree $m^{\prime}$ over $\mathbb{R}, \mathbb{R}\left(x_{1}, \ldots, x_{m^{\prime}}\right)$ is an algebraic extension of $\mathbb{R}\left(\widetilde{q}_{1}, \ldots, \widetilde{q}_{m^{\prime}}\right)$. Let $p \in \mathbb{C}\left[V_{\mathbb{R}}\right]^{K}$ be $\mathbb{R}$-valued and define $\widetilde{p} \in \mathbb{R}\left(x_{1}, \ldots, x_{m^{\prime}}\right)$ by $p(x):=p\left(x, a_{\circ}\right) . \quad \widetilde{p}$ is algebraic over $\mathbb{R}\left(\widetilde{q}_{1}, \ldots, \widetilde{q}_{m^{\prime}}\right)$ and reasoning as above shows that $p$ is algebraic over $\mathbb{R}\left(q_{1}, \ldots, q_{m^{\prime}}\right)$. Thus $\operatorname{Frac}\left(\mathbb{C}\left[V_{\mathbb{R}}\right]^{K}\right)$ is an algebraic extension of $\operatorname{Frac}\left(\tau^{*}\left(I\left[\mathfrak{k}^{*}\right]\right)\right)$. Equivalently, $\operatorname{Frac}\left(\mathbb{C}\left[V_{\mathbb{R}}\right]^{K}\right)$ is a finite extension of $\operatorname{Frac}\left(\tau^{*}\left(I\left[\mathfrak{k}^{*}\right]\right)\right.$ ) (since $\mathbb{C}\left[V_{\mathbb{R}}\right]^{K}$ is finitely generated as an algebra over $\mathbb{C}$ ).

Remark 5.1. Note that since $q_{1}, \ldots, q_{m}$ belong to $\mathbb{C}\left[V_{\mathbb{R}}\right]^{K}$, they are algebraic over $\mathbb{R}\left(q_{1}, \ldots, q_{m^{\prime}}\right)$. Since $q_{1}, \ldots, q_{m}$ are also algebraically independent, we see that $m^{\prime}=m$ in the proof of Proposition 5.4. Similar reasoning with the invariants $\mathbb{C}\left[V_{\mathbb{R}}\right]^{K}$ shows that, in general, the transcendence degree of $\operatorname{Frac}\left(\mathbb{C}\left[V_{\mathbb{R}}\right]^{K}\right)$ over $\mathbb{C}$ is exactly the minimal codimension of $K$-orbits in $V_{\mathbb{R}}$. $\tau$ is one-to-one on $K$-orbits precisely when this minimal codimension agrees with the transcendence degree of $\operatorname{Frac}\left(\tau^{*}\left(I\left[\mathfrak{k}^{*}\right]\right)\right)$ over $\mathbb{C}$.

Theorems 1.2 and 1.3 follow immediately from Propositions 5.3 and 5.4. Moreover, the arguments in this section establish the equivalence of the following conditions:

(1) $\left(K, H_{V}\right)$ is a Gelfand pair.

(2) $\operatorname{Frac}\left(\mathbb{C}\left[V_{\mathbb{R}}\right]^{K}\right)$ is a finite extension of $\operatorname{Frac}\left(\tau^{*}\left(I\left[\mathfrak{k}^{*}\right]\right)\right)$.

(3) $\operatorname{Frac}\left(\mathbb{C}\left[V_{\mathbb{R}}\right]^{K}\right)$ is algebraic over $\operatorname{Frac}\left(\tau^{*}\left(I\left[\mathfrak{k}^{*}\right]\right)\right)$.

(4) $\mathbb{C}\left[V_{\mathbb{R}}\right]^{K}$ is finitely generated as a module over $\tau^{*}\left(I\left[\mathfrak{k}^{*}\right]\right)$.

(5) $\mathcal{P} \mathcal{D}(V)^{K_{c}}$ is finitely generated as a module over $d \iota\left(\mathcal{Z U}\left(\mathfrak{k}_{c}\right)\right)$.

(6) $\tau$ is finite-to-one on $K$-orbits.

(7) For every $\xi \in \mathfrak{k}^{\perp}, \mathcal{O}_{\xi}^{G} \cap \mathfrak{k}^{\perp}$ is a finite union of $K$-orbits.

This list, together with Condition (OC) and injectivity of $\tau$ on $K$-orbits, 
supplements the list given in Theorem 1.1. Conditions (2) and (3) are equivalent because the rings involved are finitely generated. The equivalence of (4) and (5) is given in Lemma 5.2. (4) implies (2) by standard results in algebra but it is not obvious that (2) implies (4) since it is not a priori clear that $\mathbb{C}\left[V_{\mathbb{R}}\right]^{K}$ is integral over $\tau^{*}\left(I\left[\mathfrak{k}^{*}\right]\right)$. However, (2) implies that the action of $K$ on $V$ is multiplicity free and hence (5) follows from the Main Theorem part (a) in $\left[18\right.$, p.254]. Thus we also have that $\mathbb{C}\left[V_{\mathbb{R}}\right]^{K}$ is integral over $\tau^{*}\left(I\left[\mathfrak{k}^{*}\right]\right)$. In view of the connectivity result, Theorem 4.6 in [21], Condition (6) is equivalent to $\tau$ being one-to-one on $K$-orbits. Conditions (6) and (7) are equivalent as in Theorem 3.1.

Lemma 2.1 shows that three possible situations can arise when we decompose $\mathbb{C}[V]$ under the action of $K$ :

(1) $\mathbb{C}[V]$ is $K$-multiplicity free.

(2) Some $\sigma \in \widehat{K}$ occurs in $\mathbb{C}[V]$ with infinite multiplicity.

(3) Every $\sigma \in \widehat{K}$ occurs in $\mathbb{C}[V]$ with finite multiplicity but these multiplicities are unbounded.

Theorem 1.3 shows how orbit geometry distinguishes case (1) from the remaining cases. On the other hand, the moment map is necessarily one-to-one on $K$-orbits whenever it is finite-to-one. Thus, when the action of $K$ on $V$ fails to be multiplicity free, $\tau^{-1}\left(\mathcal{O}_{\alpha}^{K}\right)$ must contain infinitely many $K$-orbits for some $\alpha \in \mathfrak{k}^{*}$. Example 4.1 fits into situation (3) and illustrates this phenomenon. It is interesting that the Orbit Method can nonetheless distinguish between cases (2) and (3). We thank Friedrich Knop for showing us the proof of the following result.

Theorem 5.5. The following conditions are equivalent:

(1) Some $\sigma \in \widehat{K}$ occurs in $\mathbb{C}[V]$ with infinite multiplicity.

(2) Every $\sigma \in \widehat{K}$ occurs in $\mathbb{C}[V]$ with infinite multiplicity.

(3) There are non-constant holomorphic $K$-invariant polynomials on $V$.

(4) $\tau^{-1}(\{0\}) \neq\{0\}$.

Proof. (3) implies (2), since if $W \subset \mathbb{C}[V]$ is a $K$-irreducible subspace and $p$ is a non-constant holomorphic $K$-invariant polynomial then the representations of $K$ on the subspaces $\left\{p^{\ell} W \mid \ell \in \mathbb{Z}^{+}\right\}$are equivalent to the representation of $K$ on $W$. To see that (1) implies (3), suppose that $\mathbb{C}[V]^{K}=\mathbb{C}$ and let $\mathcal{J}_{\sigma} \subset \mathbb{C}[V]$ be the isotypic component for $\sigma \in \widehat{K}$. The "Zusatz" on page 95 of $[19]$ asserts that $\mathcal{J}_{\sigma}$ is finitely generated as a module over $\mathbb{C}[V]^{K}$. Thus, $\operatorname{dim}\left(\mathcal{J}_{\sigma}\right)<\infty$ and $\sigma$ occurs in $\mathbb{C}[V]$ with finite multiplicity. Finally, (3) and (4) are equivalent since $\tau^{-1}(\{0\}) / K$ can be identified with the categorical quotient $V / / K_{c}=\operatorname{Spec}\left(\mathbb{C}[V]^{K}\right)$. See, for example, Theorem 8.3 in $[27]$ 
concerning this identification. It follows that $\mathbb{C}[V]^{K}=\mathbb{C}$ if and only if $\{0\}$ is the only $K$-orbit in $V$ which maps to $\{0\} \subset \mathfrak{k}^{*}$ under $\tau$.

\section{Capelli actions.}

This section concerns a special class of multiplicity free actions, introduced by Howe and Umeda in [15].

Definition 6.1. We say that the action of $K$ on $V$ is a Capelli action if the map $\tau^{*}: I\left[\mathfrak{k}^{*}\right] \rightarrow \mathbb{C}\left[V_{\mathbb{R}}\right]^{K}$ is surjective.

In view of Proposition 5.3, a Capelli action is multiplicity free since in this case $\operatorname{Frac}\left(\tau^{*}\left(I\left[\mathfrak{k}^{*}\right]\right)\right)=\operatorname{Frac}\left(\mathbb{C}\left[V_{\mathbb{R}}\right]^{K}\right)$. Alternatively, Diagram (5.5) shows that for Capelli actions, $\operatorname{d\iota }\left(\mathcal{Z U}\left(\mathfrak{k}_{c}\right)\right)=\mathcal{P} \mathcal{D}(V)^{K_{c}}$. Hence $\mathcal{P} \mathcal{D}(V)^{K_{c}}$ is abelian and Theorem 1.1 shows that the action of $K$ on $V$ is multiplicity free. In [15], Howe and Umeda refer to the question "Is the mapping given by (5.4) surjective?" as the abstract Capelli problem for the complexified action of $K_{c}$ on $V$. We see that a Capelli action is one for which the abstract Capelli problem has an affirmative answer.

The subgroups $K \subset U(V)$ which act irreducibly on $V$ and yield Gelfand pairs have been classified up to conjugacy in $U(V)$. The complexification $K_{c}$ of such a group is a connected reductive algebraic group whose representation on $\mathbb{C}[V]$ is multiplicity free. Such representations were classified by Kac in $[16]$ and his list appears in $[2,3]$. In $[15]$, Howe and Umeda examined each case on the list and determined whether or not the abstract Capelli problem has an affirmative answer. The compact forms of these actions are:

$\begin{array}{lll}U(n) & \text { acting on } \mathbb{C}^{n} & \\ U(n) & \text { acting on } S^{2}\left(\mathbb{C}^{n}\right) & \text { for } n \geq 2 \\ U(n) & \text { acting on } \Lambda^{2}\left(\mathbb{C}^{n}\right) & \text { for } n \geq 2 \\ \mathbb{T} \times S O(n, \mathbb{R}) & \text { acting on } \mathbb{C}^{n} & \text { for } n \geq 3 \\ U(n) \times U(m) & \text { acting on } \mathbb{C}^{n} \otimes \mathbb{C}^{m} & \\ \mathbb{T} \times \operatorname{Sp}(n) & \text { acting on } \mathbb{C}^{2 n} & \\ U(2) \times \operatorname{Sp}(n) & \text { acting on } \mathbb{C}^{2} \otimes \mathbb{C}^{2 n} & \\ U(n) \times \operatorname{Sp}(4) & \text { acting on } \mathbb{C}^{n} \otimes \mathbb{C}^{8} & \text { for } n \geq 4 \\ \mathbb{T} \times \operatorname{Spin}(7) & \text { acting on } \mathbb{C}^{8} & \\ \mathbb{T} \times \operatorname{Spin}(10) & \text { acting on } \mathbb{C}^{16} & \\ \mathbb{T} \times G_{2} & \text { acting on } \mathbb{C}^{7} . & \end{array}$

The algebraic conditions in Section 5 place constraints on the extent to which a multiplicity free action can fail to be Capelli. Thus, although $\tau^{*}$ can fail to be surjective for a multiplicity free action, we must always have 
that $\operatorname{Frac}\left(\mathbb{C}\left[V_{\mathbb{R}}\right]^{K}\right)$ is a finite extension of $\operatorname{Frac}\left(\tau^{*}\left(I\left[\mathfrak{k}^{*}\right]\right)\right), \mathbb{C}\left[V_{\mathbb{R}}\right]^{K}$ is finitely generated as a module over $\tau^{*}\left(I\left[\mathfrak{k}^{*}\right]\right)$ and $\mathcal{P} \mathcal{D}(V)^{K_{c}}$ is finitely generated as a module over $d \iota\left(\mathcal{Z U}\left(\mathfrak{k}_{c}\right)\right)$.

In [15], the problem of describing the elements of $\mathcal{Z U}\left(\mathfrak{k}_{c}\right)$ which map to certain canonical generators for $\mathcal{P} \mathcal{D}(V)^{K_{c}}$ is referred to as the concrete Capelli problem for a Capelli action. As explained in [3], whenever $\left(K, H_{V}\right)$ is a Gelfand pair the algebra $\mathbb{C}\left[V_{\mathbb{R}}\right]^{K}$ will be a polynomial algebra

$$
\mathbb{C}\left[V_{\mathbb{R}}\right]^{K}=\mathbb{C}\left[\gamma_{1}, \ldots, \gamma_{d}\right]
$$

where the fundamental invariants $\gamma_{j}$ correspond to the $K$-irreducible components of $\mathbb{C}[V]$ with primitive highest weight vectors. Thus, the concrete Capelli problem is equivalent to finding polynomials $p_{j} \in I\left[\mathfrak{k}^{*}\right]$ with $\tau^{*}\left(p_{j}\right)=\gamma_{j}$.

\section{Examples.}

In this section, we will discuss three important Capelli actions. These are $K=U(n) \times U(m)$ acting on $V=\mathbb{C}^{n} \otimes \mathbb{C}^{m}$, and $K=U(n)$ acting on $V=S^{2}\left(\mathbb{C}^{n}\right)$ and $V=\Lambda^{2}\left(\mathbb{C}^{n}\right)$. We will see that Condition (SC) holds for $\mathbb{C}^{n} \otimes \mathbb{C}^{m}$ and $\Lambda^{2}\left(\mathbb{C}^{n}\right)$ but not for $S^{2}\left(\mathbb{C}^{n}\right)$.

As in Example 3.1, we will always identify $\mathfrak{u}(n)^{*}$ with $\mathfrak{u}(n)$ by using the Killing form $\langle A \mid B\rangle=\operatorname{tr}(A B)$ on $\mathfrak{u}(n)$. The functional corresponding to an element $A_{\theta}$ in the Lie algebra $\mathfrak{t}_{n}$ for the standard maximal torus (see Equation 3.20) is $A_{\theta^{\prime}} \mapsto\left\langle A_{\theta} \mid A_{\theta^{\prime}}\right\rangle=-\theta \cdot \theta^{\prime}$. The $\operatorname{Ad}(U(n))$-invariant polynomials on $\mathfrak{u}(n)$ are (see $[8]$ )

$$
I[\mathfrak{u}(n)]=\mathbb{C}\left[c_{1}, c_{2}, \ldots, c_{n}\right]
$$

where the Chern polynomials $c_{j}$ are defined by

$$
\operatorname{det}\left(I_{n}+\frac{\lambda}{i} A\right)=1+\sum_{j=0}^{n} c_{j}(A) \lambda^{j}
$$

Note that $c_{j}$ is real valued and homogeneous of degree $j$.

Example 7.1. Suppose that $n \geq m$ and consider the (Capelli) action of $K=U(n) \times U(m)$ on $V=\mathbb{C}^{n} \otimes \mathbb{C}^{m}$. We identify $V$ with the space $M_{n, m}$ of $n$ by $m$ complex matrices with Hermitian inner product

$$
\left\langle T_{1}, T_{2}\right\rangle=\operatorname{tr}\left(T_{1} T_{2}^{*}\right)
$$

for $T_{1}, T_{2} \in M_{n, m}$. The action of $K$ on $V$ becomes

$$
\left(k_{1}, k_{2}\right) \cdot T=k_{1} T k_{2}^{t} \quad \text { for } k_{1} \in U(n), k_{2} \in U(m) \text { and } T \in M_{n, m} .
$$


Using Equations 7.3 and 7.4 one derives the formula

$$
\tau(T)=i\left(T T^{*}, T^{t} T^{t^{*}}\right)
$$

for the moment map $\tau: V \rightarrow \mathfrak{u}(n) \oplus \mathfrak{u}(m)$.

The decomposition of $\mathbb{C}[V]$ under the action of $K$ is classical and given by

$$
\mathbb{C}[V]=\sum_{D} \overline{\sigma^{D}} \otimes \overline{\sigma^{D}}
$$

where $\sigma^{D}$ is the representation given by a Young's diagram $D$ and the sum is over all Young's diagrams with at most $m$ rows. (See for example [14].) The irreducible $\overline{\sigma^{D}} \otimes \overline{\sigma^{D}}$ in $\mathbb{C}[V]$ has highest weight vector $w_{1}^{j_{1}} w_{2}^{j_{2}} \ldots w_{m}^{j_{m}}$ where $j_{\ell}$ is the number of columns in $D$ having length $\ell$ and

$$
w_{\ell}(T):=\left|\begin{array}{ccc}
t_{11} & \cdots & t_{1 \ell} \\
\vdots & & \vdots \\
t_{\ell 1} & \cdots & t_{\ell \ell}
\end{array}\right| \quad \text { for } \ell=1, \ldots, m
$$

The primitive highest weight vectors are $w_{1}, \ldots, w_{m}$ and the associated fundamental invariants are

$$
\gamma_{\ell}(T)=\sum_{|I|=\ell=|J|}\left|\operatorname{det}\left(T_{I J}\right)\right|^{2}
$$

where the sum is over all $\ell$ by $\ell$ subdeterminants of $T$. We have

$$
\mathbb{C}\left[V_{\mathbb{R}}\right]^{K}=\mathbb{C}\left[\gamma_{1}, \ldots, \gamma_{m}\right] .
$$

The $\operatorname{Ad}(K)$-invariant polynomials on $\mathfrak{k}=\mathfrak{u}(n) \oplus \mathfrak{u}(m)$ are

$$
I[\mathfrak{k}]=I[\mathfrak{u}(n)] \otimes I[\mathfrak{u}(m)]=\mathbb{C}\left[c_{1}, \ldots, c_{n}, c_{1}^{\prime}, \ldots, c_{m}^{\prime}\right]
$$

where the $c_{j}$ 's and $c_{j}^{\prime}$ 's are the Chern polynomials on the $\mathfrak{u}(n)$ and $\mathfrak{u}(m)$ factors respectively. The classical Capelli identities correspond (via Diagram 5.5) to the following result.

Lemma 7.1. $\tau^{*}: I[\mathfrak{u}(n) \oplus \mathfrak{u}(m)] \rightarrow \mathbb{C}\left[V_{\mathbb{R}}\right]^{K}$ is given by

$$
\begin{array}{ll}
\tau^{*}\left(c_{\ell}\right)=\gamma_{\ell}=\tau^{*}\left(c_{\ell}^{\prime}\right) & \text { for } \ell=1, \ldots, m \\
\tau^{*}\left(c_{\ell}\right)=0 & \text { for } \ell=m+1, \ldots, n .
\end{array}
$$


It is not difficult to verify these identities by applying $\tau^{*}\left(c_{\ell}\right), \tau^{*}\left(c_{\ell}^{\prime}\right)$ and $\gamma_{\ell}$ to elements $T \in V$ of the form

$$
T=\left[\frac{D}{0}\right] \quad \text { where } D=\left[\begin{array}{llll}
d_{1} & & & \\
& d_{2} & & \\
& & \ddots & \\
& & & d_{m}
\end{array}\right] \text { is diagonal. }
$$

In fact, it suffices to check the identities for elements $T$ of the above form since the polynomials involved are $K$-invariant and every $K$ orbit in $V$ contains such a point. We outline a proof of this fact.

Let $T \in V$ be given and assume for simplicity that $T$ has rank $m .^{3}$ The moment map for the left action of $U(n)$ on $V$ is $\tau_{\mathfrak{u}(n)}(T)=i T T^{*}$. The spectral theorem shows that for some $k_{1} \in U(n)$ one has

$$
\tau_{\mathfrak{u}(n)}\left(k_{1} T\right)=k_{1} \tau_{\mathfrak{u}(n)}(T) k_{1}^{*}=i\left[\begin{array}{llll}
a_{1} & & & \\
& a_{2} & & \\
& & \ddots & \\
& & & a_{n}
\end{array}\right]
$$

diagonal. Since $T$ has rank $m$ we can choose $k_{1}$ so that $a_{1}, \ldots a_{m}$ are positive real numbers and $a_{m+1}=\cdots=a_{n}=0$. Thus, $k_{1} T$ has the form

$$
k_{1} T=\left[\frac{A}{0}\right]
$$

where $A$ is an $m \times m$ matrix with pair wise orthogonal rows and whose $j$ 'th row has norm $d_{j}=\sqrt{a_{j}}$. Multiplying each row of $A$ by the reciprocal of its norm yields a matrix $k_{2} \in U(m)$. One checks easily that

$$
k_{1} T k_{2}^{-1}=\left[\begin{array}{ccc}
d_{1} & & \\
& \ddots & \\
& & d_{m} \\
\hline & \bigcirc
\end{array}\right]
$$

Applying $\tau$ given by Equation 7.5 to a point $T \in V$ of the form 7.11 yields

$$
\tau\left(\left[\frac{D}{0}\right]\right)=i\left(\left[\begin{array}{cc|c|c}
\left|d_{1}\right|^{2} & & & \\
& \ddots & & \bigcirc \\
& & \left|d_{m}\right|^{2} & \\
\hline & \bigcirc & \bigcirc
\end{array}\right],\left[\begin{array}{ccc}
\left|d_{1}\right|^{2} & & \\
& \ddots & \\
& & \left|d_{m}\right|^{2}
\end{array}\right]\right)
$$

\footnotetext{
${ }^{3}$ One can remove this hypothesis without too much difficulty but in any case this is the situation generically.
} 
Since every $K$-orbit in $V$ contains such a point $T$,

$$
\tau(V) \cap \mathfrak{t}=\left\{i\left(\left[\begin{array}{l|l}
A \mid 0 \\
\hline 0 \mid 0
\end{array}\right], A\right) \mid A=\left[\begin{array}{lll}
a_{1} & & \\
& \ddots & \\
& & a_{m}
\end{array}\right], a_{1}, \ldots, a_{m} \geq 0\right\}
$$

where $\mathfrak{t}=\mathfrak{t}_{n} \oplus \mathfrak{t}_{m}$. The Weyl group acts by permutations on $a_{1}, \ldots, a_{m}$. Thus, each Weyl orbit through an integral point in $\tau(V) \cap \mathfrak{t}$ contains exactly one point of the form

$$
i\left(\left[\begin{array}{c|c}
A & 0 \\
\hline 0 & 0
\end{array}\right], A\right)
$$

with $a_{1} \geq a_{2} \geq \cdots \geq a_{m} \geq 0$ all integers. The associated representation of $K$ is $\overline{\sigma^{D}} \otimes \overline{\sigma^{D}}$ where $\mathrm{D}$ is the Young's diagram with rows of length $a_{1}, \ldots, a_{m}$. The complex conjugate $\overline{\sigma^{D}}$ of $\sigma^{D}$ appears because the pairing $\langle\cdot \mid \cdot\rangle$ used to identify $\mathfrak{t}^{*}$ with $\mathfrak{t}$ introduces a factor of $i^{2}=-1$. This shows that Condition (SC) holds for this example.

Example 7.2. The space $S^{2}\left(\mathbb{C}^{n}\right)$ of symmetric 2 -tensors on $\mathbb{C}^{n}$ can be realized as the $n$ by $n$ symmetric matrices, $V:=\left\{T \in M_{n, n} \mid T^{t}=T\right\}$ with Hermitian inner product

$$
\left\langle T_{1}, T_{2}\right\rangle=\operatorname{tr}\left(T_{1} T_{2}^{*}\right) \quad \text { for } T_{1}, T_{2} \in V .
$$

$K=U(n)$ acts unitarily on $(V,\langle\cdot, \cdot\rangle)$ via

$$
k \cdot T:=k T k^{t} .
$$

Using Equations 7.19 and 7.18, one computes the moment map for this action as

$$
\tau: V \rightarrow \mathfrak{u}(n), \quad \tau(T)=2 i T T^{*} .
$$

Since the action of $U(n)$ on $V$ is multiplicity free, we know that Condition (OC) holds. The main issue here is to demonstrate that Condition (SC) is violated for this example. The decomposition of $\mathbb{C}[V]$ under the action of $U(n)$ can be written as

$$
\mathbb{C}[V]=\sum_{\substack{D \text { with at most n rows } \\ \text { all of even length }}} \overline{\sigma^{D}} .
$$

A highest weight vector for $\overline{\sigma^{D}}$ in $\mathbb{C}[V]$ is $h_{D}:=w_{1}^{j_{1}} w_{2}^{j_{2}} \ldots w_{n}^{j_{n}}$ where $w_{\ell}$ is given by Equation 7.7 and $j_{\ell}$ is one half the number of columns in $D$ of length $\ell$. (See $[\mathbf{1 4}]$.) 
It is of course standard that one can diagonalize a symmetric bilinear form. Here we require the stronger fact that this can be done unitarily.

Proposition 7.2. For any $n \times n$ symmetric matrix $T$ with entries in $\mathbb{C}$, there is a unitary matrix $k$ with $k T k^{t}$ diagonal. Equivalently, if $B$ is a symmetric bilinear form on a complex Hermitian vector space then there is an orthonormal basis with respect to which $B$ is diagonal.

We believe that this result must be known but were unable to locate a reference. In any case, the proof involves a straightforward modification of the standard proof for diagonalization of symmetric bilinear forms and will be omitted.

Proposition 7.2 shows that each $U(n)$-orbit in $V$ contains a point of the form

$$
T=\left[\begin{array}{llll}
d_{1} & & \\
& \ddots & \\
& & d_{n}
\end{array}\right]
$$

and one has

$$
\tau(T)=2 A_{\theta}:=2 i\left[\begin{array}{llll}
\theta_{1} & & \\
& \ddots & \\
& & \theta_{n}
\end{array}\right]
$$

where $\theta_{j}:=\left|d_{j}\right|^{2}$. Conjugating by permutation matrices, we see that each $\operatorname{Ad}^{*}(U(n))$-orbit in $\tau(V)$ meets $\mathfrak{t}^{*} \cong \mathfrak{t}$ in the Weyl orbit through a point of the form $A_{\theta}$ with $\theta_{1} \geq \theta_{2} \cdots \geq \theta_{n}$. Note that $\operatorname{Ker}(T \rightarrow U(V))=\{ \pm I\}$ and hence

$$
\begin{aligned}
\operatorname{Ker}(\mathfrak{t} & \underset{\exp }{\longrightarrow} T \rightarrow U(V)) \\
& =\left\{A_{\theta} \mid 2 \theta_{1}, \ldots, 2 \theta_{n} \in \mathbb{Z}\right\} \cup\left\{A_{\theta} \mid 2 \theta_{1}, \ldots, 2 \theta_{n} \in \mathbb{Z}+\frac{1}{2}\right\} .
\end{aligned}
$$

The integral points in $\mathfrak{t}^{*} \cong \mathfrak{t}$ have the form $A_{\theta}$ where $-\theta \cdot \theta^{\prime} \in \mathbb{Z}$ for all $\theta^{\prime}$ with $\theta_{1}^{\prime}, \ldots, \theta_{n}^{\prime} \in \mathbb{Z}$ and all $\theta^{\prime}$ with $\theta_{1}^{\prime}, \ldots, \theta_{n}^{\prime} \in \mathbb{Z}+\frac{1}{2}$. Equivalently, $A_{\theta}$ is an integral point if and only if $\theta_{1}, \ldots, \theta_{n} \in \mathbb{Z}$ and $\theta_{1}+\cdots+\theta_{n}$ is even. Equation 7.23 shows that the integral orbits in $\tau(T)$ are precisely those which pass through points $A_{\theta}$ with $\theta_{1} \geq \theta_{2} \geq \cdots \geq \theta_{n} \geq 0$ all integers satisfying $\theta_{1}+\cdots+\theta_{n} \in 2 \mathbb{Z}$. The corresponding representation of $U(n)$ is the conjugate of the representation given by a Young's diagram with rows of length 
$\left(\theta_{1}, \theta_{2}, \ldots, \theta_{n}\right)$. That is, the representations that appear in $\tau(V)$ are parameterized by the Young's diagrams with even size (total number of cells). Equation 7.21 shows that of these, only the ones with each of $\theta_{1}, \theta_{2}, \ldots, \theta_{n}$ even occur in $\mathbb{C}[V]$. Thus there are infinitely many integral orbits in $\tau(V)$ that correspond to representations of $U(n)$ that don't appear in $\mathbb{C}[V]$. In particular, this example shows that we can have $\widehat{G}_{K} \varsubsetneqq\left\{\rho_{\xi} \mid \xi \in \mathfrak{k}^{\perp}\right.$ is integral $\}$ even when $\left(K, H_{V}\right)$ is a Gelfand pair. (See Proposition 4.1 and Example 4.1.)

The fundamental invariants in $\mathbb{C}\left[V_{\mathbb{R}}\right]^{U(n)}$ are $\gamma_{1}, \ldots, \gamma_{n}$ given by Equation 7.8 where the notation is as in Example 7.1. By computing $\tau^{*}\left(c_{\ell}\right)(T)$ and $\gamma_{\ell}(T)$ for a diagonal matrix $T \in V$, one shows that

$$
\tau^{*}: I[\mathfrak{u}(n)] \rightarrow \mathbb{C}[V]^{U(n)} \text { is given by } \tau^{*}\left(c_{\ell}\right)=2^{\ell} \gamma_{\ell} .
$$

It is more difficult to describe $\tau^{*}$ in the skew-symmetric case which follows.

Example 7.3. The space $\Lambda^{2}\left(\mathbb{C}^{n}\right)$ of skew-symmetric 2 -tensors on $\mathbb{C}^{n}$ can be realized as the $n$ by $n$ skew-symmetric matrices, $V:=\left\{T \in M_{n, n} \mid T^{t}=-T\right\}$ with Hermitian inner product

$$
\left\langle T_{1}, T_{2}\right\rangle=\operatorname{tr}\left(T_{1} T_{2}^{*}\right) \quad \text { for } T_{1}, T_{2} \in V
$$

$K=U(n)$ acts unitarily on $(V,\langle\cdot, \cdot\rangle)$ via

$$
k \cdot T:=k T k^{t} .
$$

As in Example 7.2 the moment map for this action is

$$
\tau: V \rightarrow \mathfrak{u}(n), \quad \tau(T)=2 i T T^{*} .
$$

Condition (OC) holds since the action of $U(n)$ on $V$ is multiplicity free. We will show that Condition (SC) holds for this example. In view of the similarity between Examples 7.2 and 7.3, this fact is somewhat curious. The decomposition of $\mathbb{C}[V]$ under the action of $U(n)$ is given by

$$
\mathbb{C}[V]=\sum_{\substack{D \text { with at most n rows and } \\ \text { all columns of even length }}} \overline{\sigma^{D}} .
$$

A highest weight vector for $\overline{\sigma^{D}}$ in $\mathbb{C}[V]$ is $h_{D}:=\nu_{1}^{j_{1}} \nu_{2}^{j_{2}} \ldots \nu_{m}^{j_{m}}$ where $m:=$ $\left\lfloor\frac{n}{2}\right\rfloor, \nu_{\ell}(T)$ is the Pfaffian of the skew-symmetric matrix given by the first $\ell$ rows and columns of $T \in V$ and $j_{\ell}$ is the number of columns of length $\ell$ in D. (See [14].)

In order to find a cross section for the $U(n)$-orbits in $V$, we require a skewsymmetric analog of Proposition 7.2. Again, the result below is standard 
if one replaces the word "unitary" by "invertible" and removes the word "orthonormal". The proof will be omitted.

Proposition 7.3 For any $n \times n$ skew-symmetric matrix $T$ with entries in $\mathbb{C}$, there is a unitary matrix $k$ with

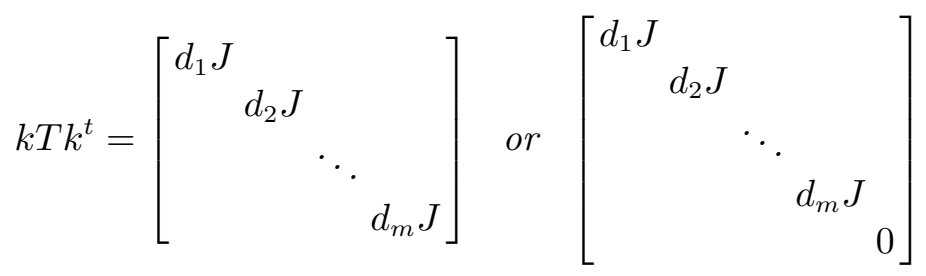

for $n=2 m$ even or $n=2 m+1$ odd respectively where $J:=\left[\begin{array}{rr}0 & 1 \\ -1 & 0\end{array}\right]$. Equivalently, if $B$ is a skew-symmetric bilinear form on a Hermitian vector space, then there is an orthonormal basis

$$
\mathcal{B}= \begin{cases}\left\{u_{1}, v_{1}, \ldots, u_{m}, v_{m}\right\} & \text { for } n=2 m \\ \left\{u_{1}, v_{1}, \ldots, u_{m}, v_{m}, w\right\} & \text { for } n=2 m+1\end{cases}
$$

with $B\left(u_{j}, v_{j}\right)=d_{j}=-B\left(v_{j}, u_{j}\right)$ and $B(\alpha, \beta)=0$ for other pairs of basis vectors from $\mathcal{B}$.

For our purposes here it makes no difference whether $n$ is even or odd. Suppose $n=2 m$ to simplify notation. Proposition 7.3 shows that each $U(n)$-orbit in $V$ contains a skew-symmetric matrix $T$ of the form

$$
T=\left[\begin{array}{llll}
d_{1} J & & & \\
& d_{2} J & & \\
& & \ddots & \\
& & & d_{m} J
\end{array}\right] .
$$

One has

$$
\tau(T)=2 i\left[\begin{array}{llllll}
\left|d_{1}\right|^{2} & & & & & \\
& \left|d_{1}\right|^{2} & & & & \\
& & \left|d_{2}\right|^{2} & & & \\
& & \left|d_{2}\right|^{2} & & & \\
& & & \ddots & & \\
& & & & \mid & \\
& & & & \left|d_{m}\right|^{2} & \\
& & & & & \left|d_{m}\right|^{2}
\end{array}\right]
$$


and one can suppose that $\left|d_{1}\right|^{2} \geq\left|d_{2}\right|^{2} \geq \cdots \geq\left|d_{m}\right|^{2} \geq 0$. In this case we have

$$
\text { (7.34) Ker }(\mathfrak{t} \underset{\exp }{\longrightarrow} T \rightarrow U(V))= \begin{cases}\left\{A_{\theta} \mid \theta_{1}, \ldots, \theta_{n} \in \mathbb{Z}\right\} & \text { for } n>2 \\ \left\{A_{\theta} \mid \theta_{1}+\theta_{2} \in \mathbb{Z}\right\} & \text { for } n=2 .\end{cases}
$$

Thus the integral points $A_{\theta}$ in $\mathfrak{t}^{*} \cong \mathfrak{t}$ have $\theta_{1}, \ldots, \theta_{n}$ all integers. Also we must have $\theta_{1}=\theta_{2}$ when $n=2$. We see that each integral orbit in the image of the moment map passes through a point $A_{\theta} \in \mathfrak{t}$ with $\theta_{1}=\theta_{2} \geq \theta_{3}=\theta_{4} \geq$ $\cdots \geq \theta_{n-1}=\theta_{n} \geq 0$ all integers. The corresponding representation of $U(n)$ is conjugate to $\sigma^{D}$ where $D$ is a Young's diagram where pairs of successive rows have the same length. Equivalently, all columns of $D$ have even length. These are the representations appearing in Equation 7.29 so Condition (SC) holds here.

\section{References}

[1] C. Benson, J. Jenkins, R. Lipsman and G. Ratcliff, The moment map for a multiplicity free action, Bull. Amer. Math. Soc., 31 (1994), 185-190.

[2] C. Benson, J. Jenkins and G. Ratcliff, On Gelfand pairs associated with solvable Lie groups, Trans. Amer. Math. Soc., 321 (1990), 85-116.

[3] , Bounded K-spherical functions on Heisenberg groups, J. Functional Analysis, 105 (1992), 409-443.

[4] G. Carcanno, A commutativity condition for algebras of invariant functions, Boll. Un. Mat. Italiano, 7 (1987), 1091-1105.

[5] L. Corwin and F. Greenleaf, Complex algebraic geometry and calculations of multiplicities for induced representations of nilpotent Lie groups, Trans. Amer. Math. Soc., 305 (1988), 601-622.

[6] Spectrum and multiplicities for restrictions of unitary representations in nilpotent Lie groups, Pacific J. Math., 135 (1988), 233-267.

[7] L. Corwin and F. Greenleaf (with G. Grélaud), Direct integral decompositions and multiplicities for induced representations of nilpotent Lie groups, Trans. Amer. Math. Soc., 304 (1987), 549-583.

[8] J. Dupont, Curvature and Characteristic Classes, Lecture Notes in Math., SpringerVerlag, New York, 640 (1978).

[9] I.M. Gelfand, Spherical functions on symmetric spaces, Dokl. Akad. Nauk USSR, 70 (1950), 5-8, Amer. Math. Soc. Transl., 37 (1964), 39-44.

[10] V. Guillemin and S. Sternberg, Geometric quantization and multiplicities of group representations, Inventiones Math., 67 (1982), 515-538.

[11]_, Multiplicity free spaces, J. Differential Geom., 19 (1984), 31-56.

[12] G.J. Heckman, Projections of orbits and asymptotic behavior of multiplicities for compact connected Lie groups, Inventiones Math., 67 (1982), 333-356.

[13] S. Helgason, Groups and Geometric Analysis, Academic Press, New York, 1984.

[14] R. Howe, Remarks on classical invariant theory, Trans. Amer. Math. Soc., 313 (1989), 539-570.

[15] R. Howe and T. Umeda, The Capelli identity, the double commutant theorem and multiplicity-free actions, Math. Ann., 290 (1991), 565-619. 
[16] V. Kac, Some remarks on nilpotent orbits, Journal of Algebra, 64 (1980), 190-213.

[17] A.A. Kirillov, Unitary reoresentations of nilpotent Lie groups, Uspeki Mat. Nauk, 17 (1962), 57-110, Russian Math. Surveys, 17 (1962), 57-110.

[18] F. Knop, A Harish-Chandra homomorphism for reductive group actions, Annals of Math., 140 (1994), 253-289.

[19] H. Kraft, Geometrische Methoden in der Invariantentheorie, Aspekte der Mathematik. Vieweg, Braunschweig, 1985.

[20] M. Krämer, Sphärische untergruppen in kompakten zusammenhängenden Lie-gruppen, Compos. Math., 38 (1979), 129-153.

[21] E. Lerman, R. Montgomery and R. Sjamaar, Examples of singular reduction, Symplectic Geometry, 192, London Math. Soc. Lecture Notes, Cambridge U. Press, (1993), 127-155.

[22] R. Lipsman, The Penny-Fuijawara Placherel formula for Gelfand pairs, Rocky Mountain J. Math., 26 (1996), 655-677.

[23] Group Representations, Lecture Notes in Math., Springer-Verlag, New York, 388 (1974).

[24] Orbit theory and harmonic analysis on Lie groups with co-compact nilradical, J. Math. Pure and Appl., 59 (1980), 337-374.

[25] Orbital parameters for induced and restricted representations, Trans. Amer. Math. Soc., 313 (1989), 433-473.

[26] , Restricting representations of completely solvable Lie groups, Canadian Journal of Math., 42 (1990), 790-824.

[27] D. Mumford, J. Fogarty and F. Kirwan, Geometric Invariant Theory, SpringerVerlag, Berlin, third edition, 1994.

[28] A.L. Onishchik and E.B. Vinberg, Lie Groups and Algebraic Groups, Springer Verlag, New York, 1990.

[29] N. Wildberger, The moment map for a Lie group representation, Trans. Amer. Math. Soc., 330 (1992), 257-268.

Received May 15, 1994 and revised 12/10/94. All authors were supported in part by the National Science Foundation.

UNIV OF Missouri-St. LouIS

ST. LouIs, MO 63121

E-mail address: benson@arch.umsl.edu

AND

SUNY AT ALBANY

Albany, NY 12222

E-mail address: jwj71@math.albany.edu

AND

UNIV OF MARYLAND

College PARK, MD 20742

E-mail address: rll@clio.umd.edu

AND

Univ of Missouri-St. Louis

ST. Louis, MO 63121

E-mail address: ratcliff@arch.umsl.edu 